\title{
Supercement for Annular Seal and Long-Term Integrity in Deep, Hot Wells "DeepTrek"
}

Phase II Annual Report

Kevin D. Edgley

Fred L. Sabins

Larry T. Watters

Issued August 31, 2005

DOE Award Number

DE-FC26-02NT41836

Submitted by

CSI Technologies, LLC 2202 Oil Center Court

Houston, TX 77073 


\section{(영 CSI Technologies}

\section{DeepTrek Project Annual Report Phase I - October 2003 through December 2004}

\section{Disclaimer}

This report was prepared as an account of work sponsored by an agency of the United States Government. Neither the United States Government, nor any agency thereof, nor any of their employees, makes any warranty, express or implied, or assumes any legal liability or responsibility for the accuracy, completeness, or usefulness of any information, apparatus, product, or process disclosed, or represents that its use would not infringe privately owned rights. Reference herein to any specific commercial product, process, or service by trade name, trademark, manufacturer, or otherwise does not necessarily constitute or imply its endorsement, recommendation, or favoring by the United States Government or any agency thereof. The views and opinions of authors expressed herein do not necessarily state or reflect those of the United States Government or any agency thereof. 


\title{
(8S) CSI Technologies
}

\section{DeepTrek Project Annual Report Phase II - January 2005 through May 2006}

\begin{abstract}
The purpose of this project is to formulate a "Supercement" designed for improving the longterm sealing integrity in HPHT wells. Phase I concentrated on chemistry studies and screening tests to design and evaluate Portland-based, hybrid Portland, and non-Portland-based cement systems suitable for further scale-up testing. Phase II work concentrated on additional lab and field testing to reduce the candidate materials list to two systems, as well as scaleup activities aimed at verifying performance at the field scale. Phase II was extended thorough a proposal to develop additional testing capabilities aimed at quantifying cementing material properties and performance that were previously not possible.

Two materials are being taken into Phase III for field testing and commercialization:

- Highly-expansive cement (Portland-based), patent pending as "Pre-Stressed Cement"

- Epoxy Resin (non-Portland-based), patent pending

In Phase II, significant effort was expended on scaling up the processes for handling resin in the field, as it is quite different than conventional Portland-based cements in mixing, personnel protection, and cleanup. Through this effort, over fifty (50) field jobs were done at a variety of temperatures and depths, most with excellent results.

Large-scale field testing was less relevant with Pre-stressed Cement, because the materials and surface processes do not vary from those that have been developed for conventional Portland materials over the last eighty (80) years. The formulation is quite unique, however, and performs very differently than conventional Portland cements downhole.
\end{abstract}




\section{Table of Contents}

Disclaimer i

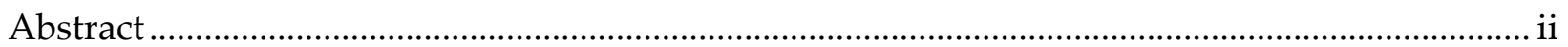

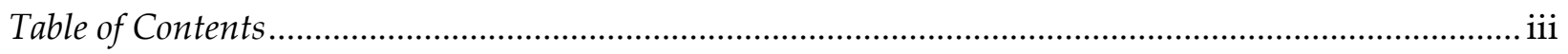

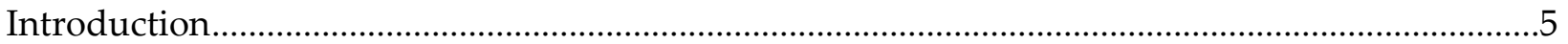

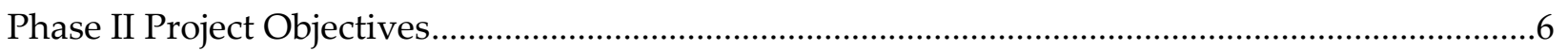

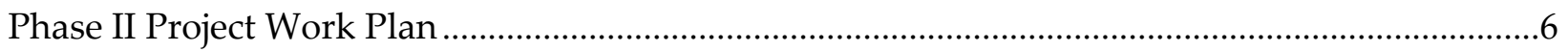

Phase II-Identification and Evaluation of Materials .................................................................6

Task 1: Manufacture Supercement to specification ...................................................................6

Task 2: Perform conventional and nonconventional batch testing to confirm the product's

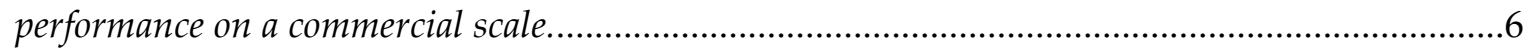

Task 3: Evaluate the product's performance in large-scale mixing, shearing, and drillout studies..6

Task 4: Test the composion's performance in a research test well.................................................6

Task 5: Develop Apparatus and Conduct tests for HPHT Annular Seal, Direct-pull tensile test,

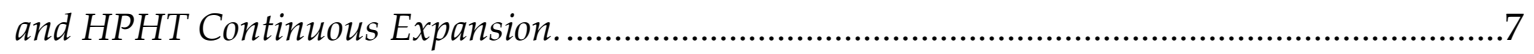

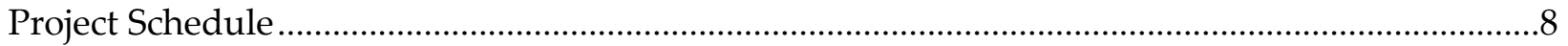

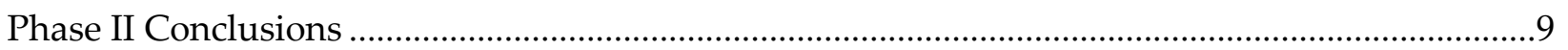

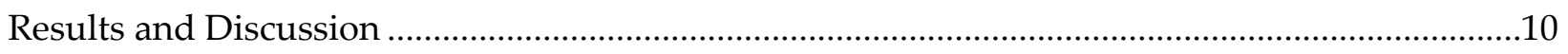

Task 1 - Manufacture Supercement to Specification........................................................................10

Task 2 - Perform conventional and unconventional batch testing to confirm the product's

performance on a commercial scale

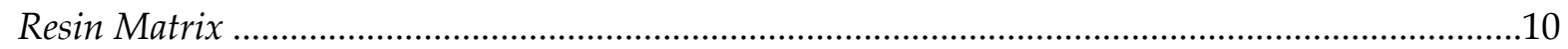

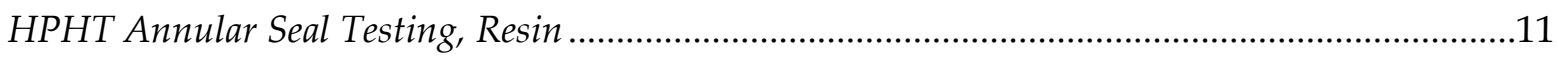

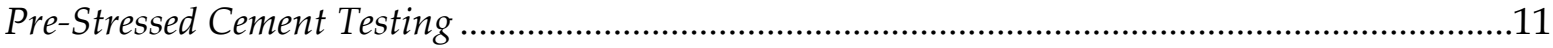

Pre-Stressed Cement Expansion Theory ........................................................................................12

Figure 1 - Stress State due to Internal Wellbore Pressure ........................................................12

Figure 2 - Initial Pre-Stressed State, Expanding Cement ...........................................................13

Figure 3 - Stress State in Pre-Stressed Cement under Load .....................................................13

Table 1 - Initial Compressive Preload, Pre-Stressed Expanding Cement......................................14

Pre-Stressed Cement HPHT Annular Seal Testing.........................................................................

Figure 4 - HPHT Annular Seal Test Schematic ....................................................................16

Table 2 - Results of Hard Formation HPHT Annular Seal Testing ............................................17

Mechanical Shear Bond Testing.................................................................................................17

Figure 5 - Mechanical Shear Bond Test Setup - Push from Top ...............................................18

Figure 6 - Mechanical Shear Bond Test Setup - Push from Bottom .............................................18 
Figure 7 - Pre-Stressed cement - Pipe end weld broke before cement-pipe shear bond..................19

Figure 8 - Failed Shearbond Test ..........................................................................................19

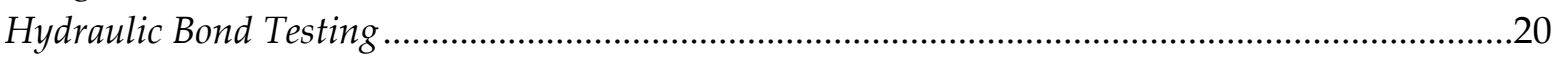

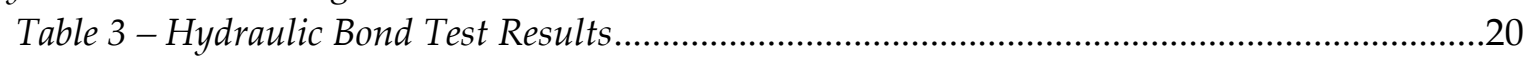

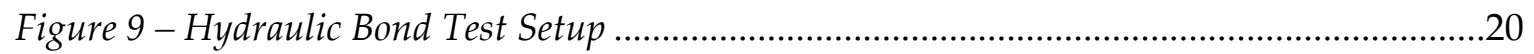

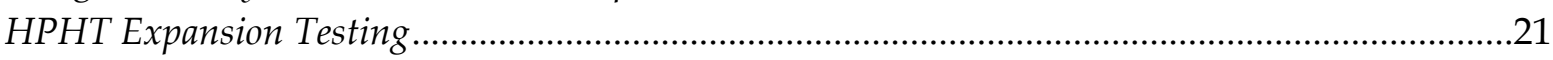

Pre-Stressed Cement HPHT Mud Channel Testing.....................................................................21

Figure 10 - Cut section of Mud Channel Simulation Test Apparatus .........................................22

Figure 11 - Closeup - Cut section of Mud Channel Simulation Test Apparatus..........................22

Task 3 - Evaluate the product's performance in large-scale mixing, shearing, and drillout

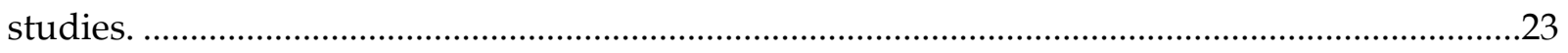

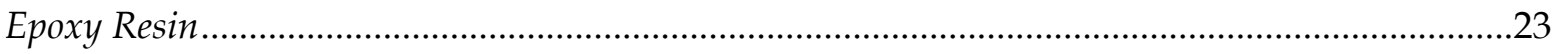

Figure 12 - Manually Introducing Resin into the Mixing Tank ..............................................23

Figure 13 - Manually Introducing Activator into the Mixing Tank ..........................................24

Figure 14 - Resin / Activator Mixture in the Mixing Tank .......................................................24

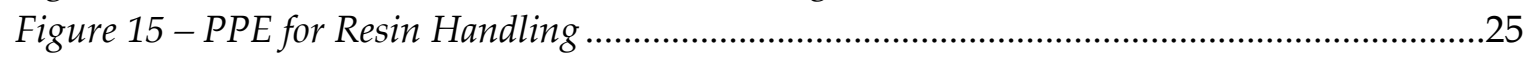

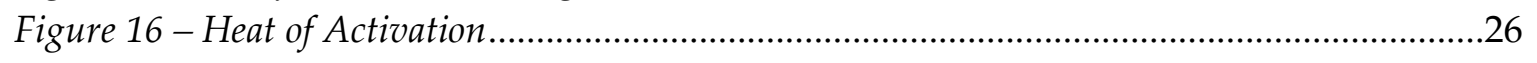

Figure 17 - Milled Resin Sample .........................................................................................27

Figure 18 - Milled Resin Sample .......................................................................................27

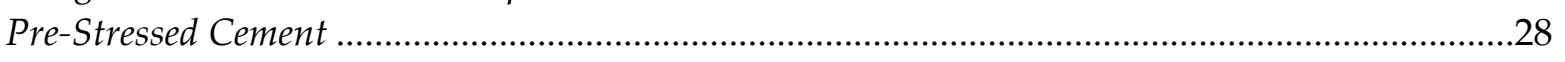

Task 4 - Test the composion's performance in a research test well ..........................................28

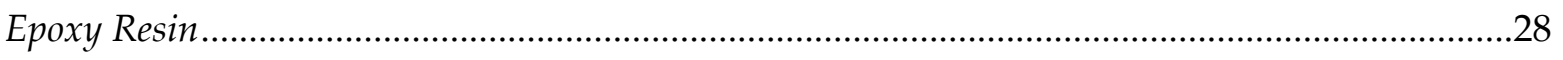

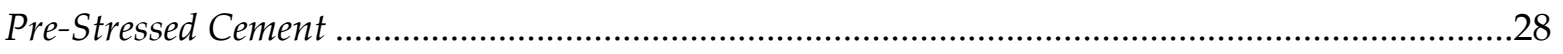

Task 5 - Phase for-cost extension - Develop HPHT Annular Seal, Direct Tensile, and HPHT

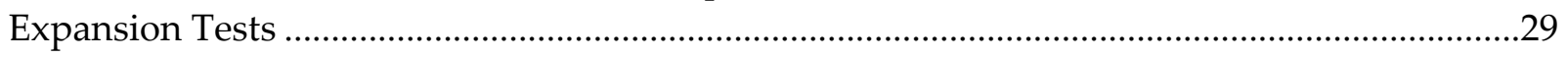

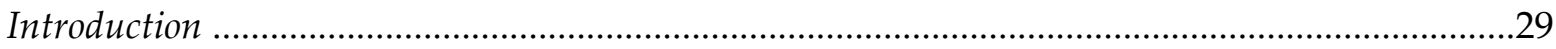

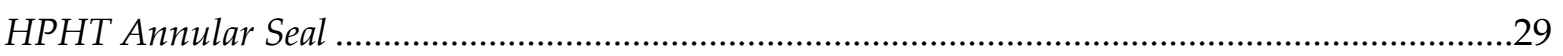

Figure 19 - HPHT Annular Seal Apparatus...........................................................................29

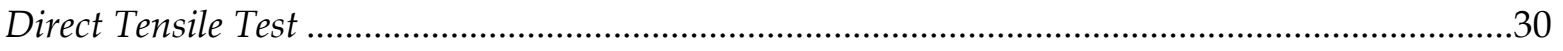

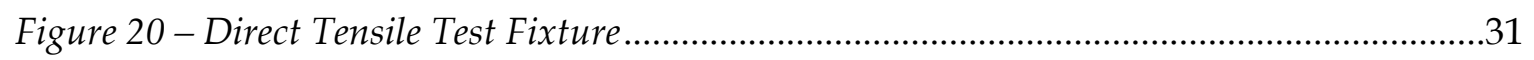

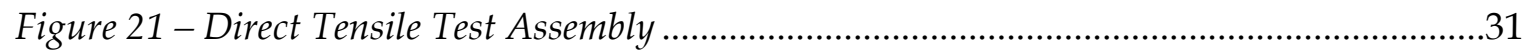

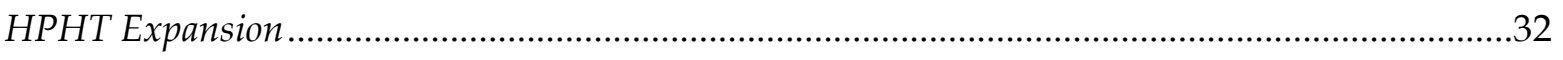

Appendix A - Resin Design Matrix and Thickening Time Tests.........................................................33

Appendix B -Thickening Time Tests on Pre-Stressed Cement .............................................................40

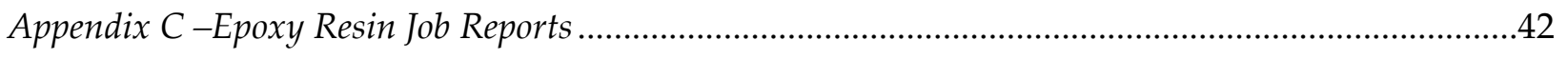

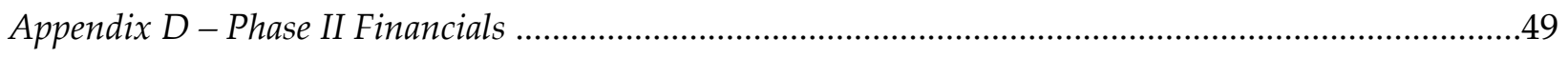

DE-FC26-02NT41438 Annual Report $\quad$ iv 


\section{(d) CSI Technologies}

\section{Introduction}

With current completion technology, oil and gas wells completed in high pressure and high temperature (HPHT) environments often experience escalating costs over the life of the well due to loss of sealant intergrity. High temperatures are generally defined as those in excess of $350 \mathrm{deg}$ F, and high pressures are those in excess of 15,000 psi. In this context, these costs are related to both the loss of production as a result of annular seal failure and the resulting remedial repair. Remedial procedures for restoring seal integrity are expensive and are often repeated several times during the life of the well. In extreme conditions, loss of annular seal can result in well abandonment and potential environmental and safety issues.

This project, as part of the Department of Energy (DOE) Deeptrek project, focuses on improving the economics associated with drilling deep, hot wells by developing new cementing materials engineered to provide long-term annular sealing in high-stress environments.

The project encompasses:

- Literature search

- Chemical cement design

- Lab screening testing

- Testing development

- Manufacturing and mixing scale-up

- Full-scale test applications and evaluations

This report details the second phase results of this three-year program. Phase II work concentrated on reducing the number of candidate sealing systems generated in Phase I to the best two to be field tested in Phase III. This culling process was accomplished through laboratory testing, scaleup testing, and limited research field tests. 


\section{(48) CSI Technologies}

\section{Phase II Project Objectives}

The second phase of this project concentrated on additional laboratory test work, scaleup activities, and the development of new testing equipment and protocols. These activities served to reduce the number of candidate materials identified in Phase I to those with the best chance of meeting the project goals. At the end of Phase II, two materials remain for full field testing, economic evaluation, and commercialization in Phase III.

\section{Phase II Project Work Plan}

\section{Phase II-Identification and Evaluation of Materials}

Task 1: Manufacture Supercement to specification

- Manufacture the Supercement in a pilot plant to assess its performance on a larger scale

- Manufacture the Supercement in actual industrial quantities at a full-scale facility to finalize the manufacturing method that will be used to produce the cement commercially.

Task 2: Perform conventional and nonconventional batch testing to confirm the product's performance on a commercial scale.

- Perform standard laboratory testing of the material manufactured in Task 1 to assess the effectiveness of the compositions for field applications.

- Tests used for this task will be the same as those used in the laboratory testing of selected compostions in Phase 1, Task 3.

Task 3: Evaluate the product's performance in large-scale mixing, shearing, and drillout studies.

- Blend and mix field-application-size batches of the material with oilfield blending and mixing equipment.

- Perform laboratory tests to confirm the performance of the blended composition.

- Shear the material through a pipe loop to simulate placement in a well.

- Perform laboratory tests on the slurry to reconfirm performance after shearing.

- Create large-scale drilling targets to determine the rate of penetration with standard drilling equipment.

- Develop procedures for blending, mixing, placement, and drillout.

Task 4: Test the composion's performance in a research test well.

- Design a cement slurry as appropriate for the well conditions.

- Blend, mix, and pump the slurry into the test well.

- Conduct a post-job evaluation of the set slurry using logging results and pressure tests.

o Report on the effectiveness of binders at high temperatures and determine the best candidates for continued evaluation in Phase II. 


\section{(48) CSI Technologies}

Task 5: Develop Apparatus and Conduct tests for HPHT Annular Seal, Direct-pull tensile test, and HPHT Continuous Expansion.

- Through an approved project extension associated with Phase II, apparatus were developed to measure various performance and properties of cementing materials. Before this extension, these apparatus were not available.

o HPHT Annular Seal - take protocol developed at ambient pressure and temperature to evaluate the annular sealing performance of various cement materials at high temperature and pressure.

o HPHT Expansion - measure continuously the expansion or contraction of cement during cure and post-cure.

o Direct-pull Tensile Test - Economically measure the tensile strength of cement in a direct-pull method, in a compression test machine. 


\section{(영 CSI Technologies}

\section{Project Schedule}

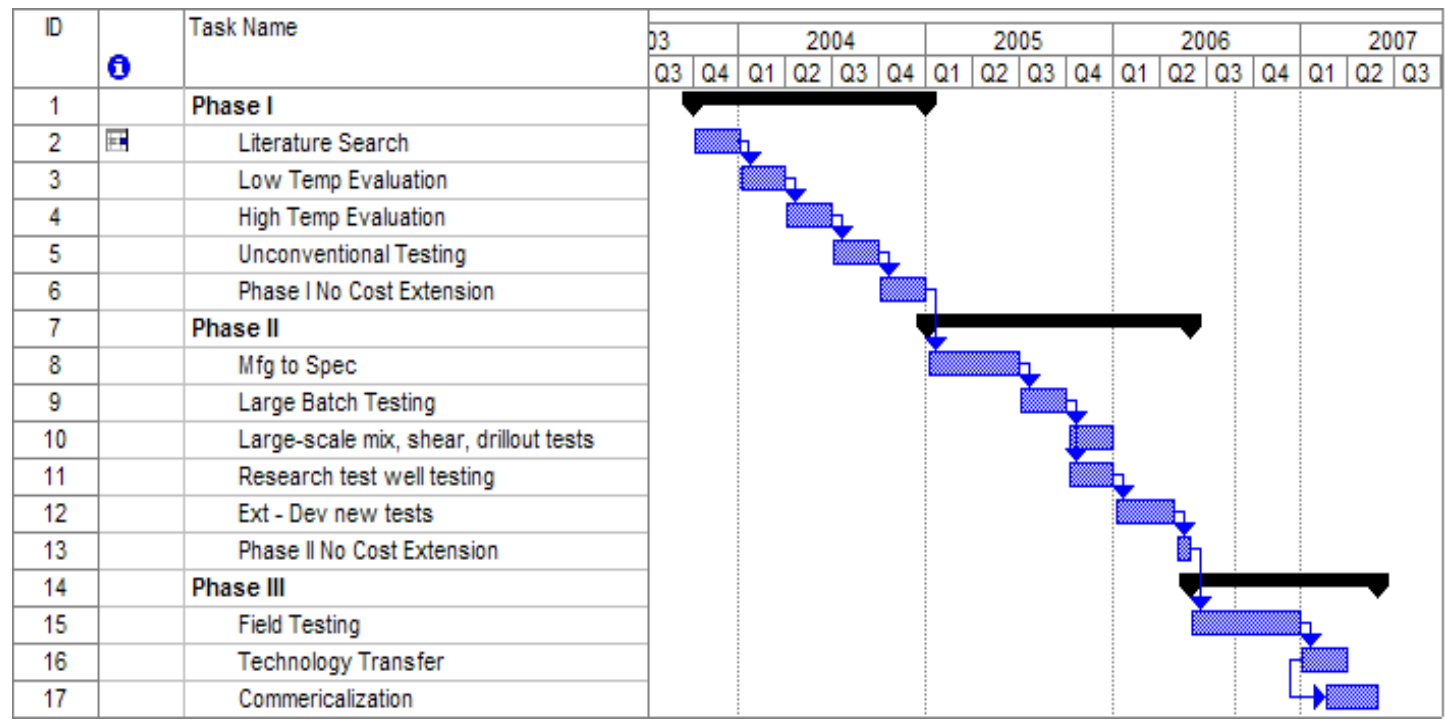




\section{(BS) CSI Technologies}

\section{Phase II Conclusions}

1. The original slate of 10 candidate sealants was reduced to two systems for Field Evaluation in Phase III. These systems are a non-shrinking Epoxy Resin and a highlyexpansive Portland cement slurry design ("Pre-Stressed Cement"). Patents are pending on both products.

2. Both sealants are controllable through a wide range of temperature conditions, making them viable materials for wellbore sealants at high termperatures.

3. Epoxy Resin is believed to seal through a different mechanism than conventional cements, relying on mechanical means and the compliant nature of the Epoxy Resin material, rather than inherent matrix strength and chemical bonding. Multiple field trials have proven the ability of the material to seal in conditions under which conventional Portland cements had repeatedly failed.

4. Pre-Stressed Cement has exhibited significantly better performance in the lab than conventional Portland Cements, due to the highly-expansive nature of the material. When cured under confined conditions, the expansion creates an internal compressive preload that enables the material to better resist induced tensile stresses in the well than conventional cements. Additionally, the material exhibits very high mechanical shear bond and hydraulic bond.

5. Through the Phase II Extension phase, tests were developed to measure various performance and property characteristics. The tests have been successful to varying degrees:

- Measure the annular sealing potential of various sealants under High Pressure and Temperature conditions.

- Measure the expansion / contraction of cement continuously at High Pressure and Temperature conditions, during and after hydration.

- Measure the tensile strength (and tensile fatigue characteristics and tensile Young's Modulus, if desired) of sealants, using a direct pull method and conventional compressive test machine. 


\section{(영 CSI Technologies}

\section{Results and Discussion}

\section{Task 1 - Manufacture Supercement to Specification}

The intent of this task was to manufacture new cementing materials in large batches to confirm that the materials are viable in manufacturing quantities. This work was initially envisioned to be applicable to newly-developed materials that have not been used in oilfield quantities in the past.

Of the two candidate materials, the Epoxy Resin is the only one for which this task is relevant, although previous non-oilfield applications for the material made this a low-risk issue. This particular material was "discovered" as an insulating material used in electric motor windings. In this application, its non-shrinking characteristic and resistance to high heat levels made it interesting as an oilfield sealant. Additionally, manufacturing capacity was already developed.

To produce the material in the quantities and formulations required for oilfield usage, CSI partnered with M\&D Industries of Lafayette, LA as a manufacturing and distribution agent. In this role, M\&D acquires the raw material, blends as required and packages the product for oilfield distribution and use. This relationship has been positive, and M\&D has effectively handled the materials. Performance and QC testing at CSI has confirmed that the material in manufactured quantities performs in a predictable, controllable, and consistent manner when compared with the initial laboratory trials.

The Pre-Stressed cement utilizes conventional Portland cements as well as conventional admixtures utilized in the oilfield cementing industry for a number of years, but in unconventional proportions. As such, no significant work was required to develop manufacturing capabilities, nor to verify performance of materials produced in large quantities.

\section{Task 2 - Perform conventional and unconventional batch testing to confirm the product's performance on a commercial scale}

\section{Resin Matrix}

Testing was completed in a program designed to create a design matrix for various well conditions, up to $240 \mathrm{deg}$ F. This matrix is being codified in a database application to assist field personnel in the selection of appropriate formulations for given well conditions. The matrix was developed by manipulating the various constituents of the resin product, performing semiqualitative pump time tests, and finally quantitative thickening time and rheology tests. The pump time of the resin product, unlike Portland cement, has been found to be relatively insensitive to pressure and weighting materials, making the design task somewhat simpler than for conventional Portland cements. Resin constituents used to date produce monoliths with consistency ranging from hard plastic to stiff rubber. Different hardeners and diluents are available to push the temperature higher, and work continues to extend the matrix to higher temperatures. Generally, with the components currently in use, the material achieves a softer set at higher temperatures, meaning that the product achieves more of a rubbery consistency as 


\section{CSI Technologies}

opposed to a hard plastic. This can be a benefit to sealing performance over the life of a well, because the material is able to maintain a seal under various stress- and movement-inducing intervention activities better than a brittle material.

Appendix A contains the resin design matrix in its current form as of the date of this report up to 240 degrees. Additionally, two thickening time and rheology tests are included at 340 deg F.

\section{HPHT Annular Seal Testing, Resin}

The HPHT Annular Seal testing with resin were unsuccessful, exhibiting leakage between the resin mass and the boundary sleeve immediately upon application of differential pressure after curing. We believe that the mechanism of sealing with resin is completely different than conventional cements, and are designing modifications to the apparatus to prove or disprove that theory. Failure of the material in the lab compared with success in the field tends to support the supposition that we are not adequately simulating the field situation for this material. In the well, there are many discontinuities in the tubulars due to casing collars, and in the formation due to irregularities in the drilled rock. We believe that the compliant resin mechanically locks into these discontinuities and seals via a pressure-activated mechanical interference (much like an o-ring) rather than through chemical bonding to the steel. Further supporting this theory are the following observations from the laboratory and field work:

- Mechanical shear bond is both high and constant, and seems to be due to the material folding up and mechanically resisting the imposed motion of the tubular. In other words, the product deforms and binds up the pipe, inhibiting movement. In conventional and Pre-Stressed cements, load typically increases to a maximum at the point of bond release, followed by lower forces required to move the pipe. With resin, the force is high and constant, even after the initiation of movement.

- Relatively low hydraulic bond in smooth-bore test pipes, except when utilized with another material, such as Ultra Spacer, a CSI fluid product that is very effective in blocking flow paths. Hydraulic bond values reported in this report are with the Ultra Spacer product.

- Over 50 jobs placed in the field have effectively shut off gas and other wellbore fluid flows with a high success rate.

To test the theory, new sleeves and loading tubes will be made with discontinuities in the inner surface of the boundary sleeve and the outer surface of the loading tube to simulate the casing collar irregularities present in the wellbore. These will be used in place of the smooth machined parts used to date, and we expect improved performance in the lab test.

\section{Pre-Stressed Cement Testing}

Pre-Stressed conventional cement testing focused on conventional cement lab tests, such as rheology, thickening time, fluid loss, etc. Unconventional testing involved HPHT Annular seal and HPHT expansion testing utilizing equipment developed during the extension phase of the 


\section{(Bis) CSI Technologies}

project. Another test focused on the limits of confinement to address concerns that less than $100 \%$ fill conditions in the cemented annulus would cause the material to produce a friable matrix. Because the material requires some level of confinement to function, fatigue characteristics and tensile and compressive strengths have been difficult to obtain.

\section{Pre-Stressed Cement Expansion Theory}

Pre-Stressed cement functions by expanding against confinement during the hydration process. Generally higher levels of confinement (hard formations or pipe-in-pipe conditions) result in the highest level of prestress. In practice, as the cement tries to expand against the confinement, significant levels of compressive stress build up within the cement matrix as it cures. Some confinement is critical to the function of the product; in the absence of confinement the internal stresses destroy the matrix during hydration. Because cement is generally strong in compression and weak in tension, this high initial compressive stress "pre-stresses" the cement much like steel reinforcing rods do in construction concrete. When wellbore tubulars are pressurized, a triaxial stress state is produced in the cement matrix, consisting of a compressive radial component, a hoop (tangential) tensile component, and a shear axial component. By preloading in compression, the cement has a higher resistance to induced tensile hoop stresses than do conventional non-expanding cements.

Figure 1 shows, in a horizontal plane cut through the wellbore, an infinitesimally small portion of cement and the stresses imposed upon it by internal pressure in the wellbore tubulars.

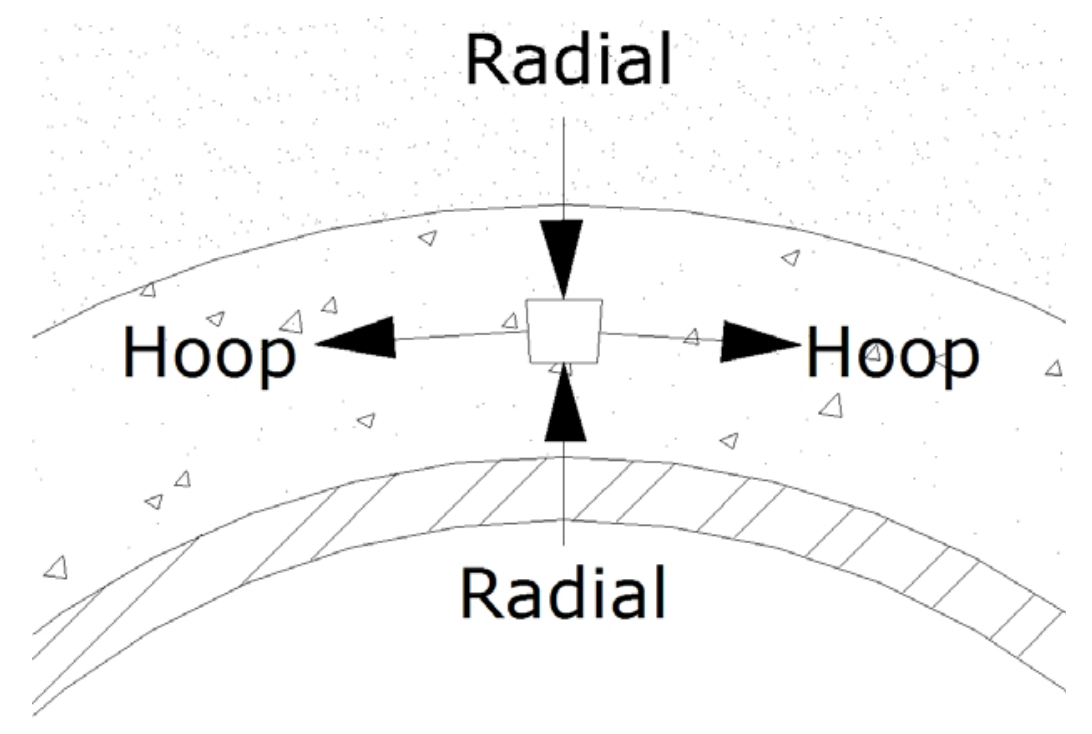

Figure 1 - Stress State due to Internal Wellbore Pressure

Obviously, if the induced tensile stress becomes higher than the tensile strength of the material, a vertical crack will form in the material perpendicular to the direction of the imposed stress. 


\section{(영 CSI Technologies}

This crack comprises a leakage path through which gas or other wellbore fluids can traverse the cement sheath.

Pre-Stressed Cement, expanding against confinement in the wellbore, creates an internal compressive preload as illustrated in Figure 2.

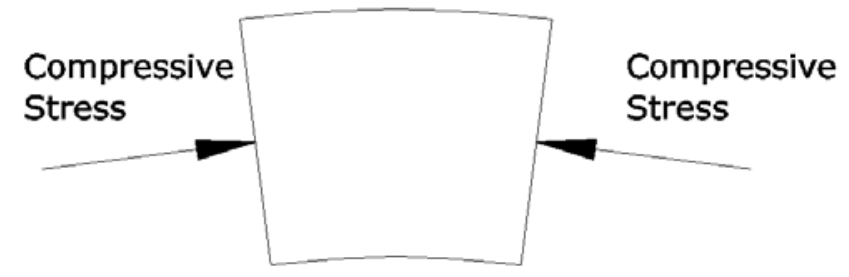

Expanding Cement

Post Cure

Figure 2 - Initial Pre-Stressed State, Expanding Cement

When pressure is applied to the wellbore tubulars, the tensile stress generated serves first to reduce the compressive prestress present in the cement before the material realizes a net tensile stress. Therefore, the effective compressive strength of the material is increased by the compressive preload applied (Figure 3, with radial compressive stress not shown).

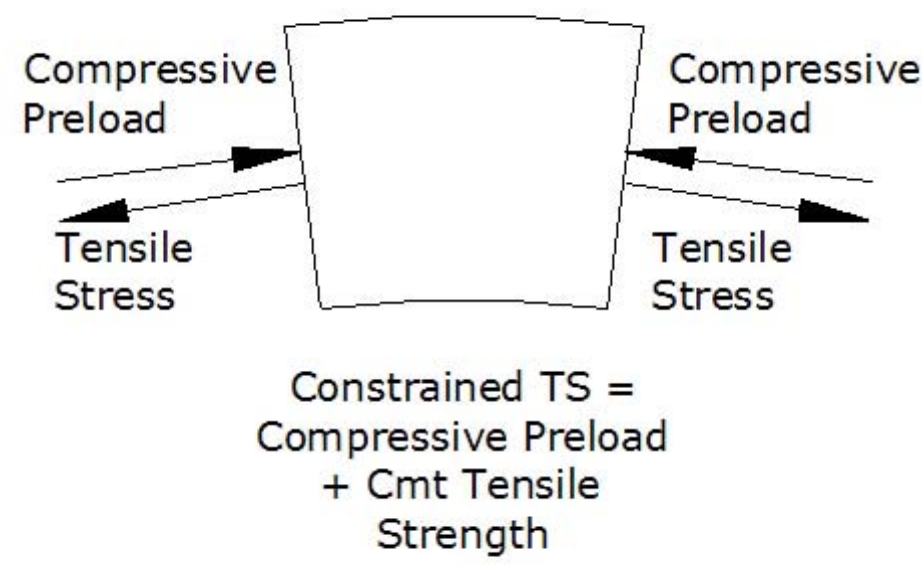

Figure 3 - Stress State in Pre-Stressed Cement under Load

Conventional non-expanding or shrinking cements must bear the induced tensile stresses within the matrix and the native tensile strength of the material. As cement is inherently weak 
in tension, conventional cements fail more quickly due to imposed tensile stresses than do PreStressed cements in annular seal performance testing.

HTHP Annular Seal testing utilized steel, thinwall aluminum, and thinwall PEEK plastic sleeves to simulate the confinement presented in the wellbore by strong, intermediate, and weak formations respectively. The estimated effective initial compressive prestress (which must be overcome before the cement experiences tensile stresses due to wellbore tubular pressurization) for each condition is presented in Table 1 . The data in Table 1 is calculated by measurements of the diametral change in the sleeve materials after curing and testing in the HTHP Annular Seal apparatus. FEA simulations were run with various loading values to determine internal pressures required in a sleeve to produce the displacements observed. As indicated in Figure 3 above, the Initial Compressive Prestress effectively increases the material's tensile strength by the same amount.

\begin{tabular}{|l|c|}
\hline Sleeve Material (Formation Type) & Estimated Effective Initial Compressive Prestress (psi) \\
\hline Steel (Strong) & 3,290 \\
\hline Thinwall Aluminum (Intermediate) & 2,410 \\
\hline Thinwall PEEK Plastic (Weak) & 1,900 \\
\hline
\end{tabular}

Table 1 - Initial Compressive Preload, Pre-Stressed Expanding Cement

\section{Pre-Stressed Cement HPHT Annular Seal Testing}

Pre-Stressed cement showed very promising results when compared with the Baseline 99 cement system (Portland cement, retarded, with 35\% silica) in the strong formation case, although the weak formation results showed characteristics consistent with previous experiences with expanding cement. Tests with intermediate formations are ongoing, but appear positive so far. In the weak formation case, previous experience with expanding cement has shown a tendency of the cement to pull away from the inner pipe as it expands against the softer formation. In the lab, this occurred in the test cell, and leakage was immediate around the inner pipe. As in the discussion on resin, this may be mitigated by introducing discontinuities on the inner and outer pipes to simulate the effect of casing joints and hole irregularities.

The test apparatus design is detailed under Task 5 discussion. Operation of the test is designed to allow cement to cure in the test fixture under pressure and temperature conditions, and then to impose a small differential pressure across the short (approximately 3.5" long) cement mass. Pressure is then applied to the central loading tube, which imposes compressive and tensile stresses in the cement mass. Test procedure consists of (refer to Figure 4, HPHT Annular Seal Test Schematic to understand references to various components in the test setup): 


\section{(BS) CSI Technologies}

1. Make up the boundary sleeve in the bottom flange, with the central loading tube bolted to the bottom flange. Bolt the bottom flange to the body.

2. Pour approximately 1 " of sand in the bottom of the boundary sleeve and saturate the sand with water.

3. Pour cement on top of the saturated sand pack.

4. Pour water in the annulus between the boundary sleeve and the fixture ID, and continue on top of the cement until the fixture is full.

5. Bolt on the top cap and place the assembly in the oven.

6. Connect the lines as shown in the test schematic.

7. Open the LP Shutoff, HP Shutoff, and Equalization Valves

8. Close the Release and Bleed Valves.

9. Apply system pressure with the Low Pressure Regulator. Because of the equalization line, the same pressure will be applied throughout the fixture interior, including below the cement.

10. After curing is complete, close the equalization valve and adjust the High Pressure Regulator until approximately $10-20$ psi is present on the Differential Pressure Gage.

11. Load the cement by pressurizing the central loading tube with mineral oil, alternately pressurizing for one minute, then releasing the pressure through the release valve for one minute. The use of mineral oil prevents vaporization of the loading fluid at test temperature when the pressure is reduced to atmospheric. The bleed valve is used to make sure the lines are purged of air before testing starts.

12. Loading protocol is as follows:

a. Pressurize to 1,000 psi for one minute, release to 0 psi for one minute. Check the Differential Pressure Gage to determine if differential pressure is still present. When the cement fails, the gage will go to 0 .

b. Pressurize to 2,000 psi for one minute, release to 0 psi for one minute. Check the Differential Pressure Gage as before.

c. Continue steps a and b in increments of 1,000 psi, until 10,000 psi is reached, while monitoring the Differential Pressure Gage. After 10,000 psi is applied and released, repeat the loading protocol starting at 1,000 psi.

d. After 10 cycles are successfully attained, continue loading directly to 10,000 psi until the differential pressure gage reads 0 .

13. After the test is complete, cool the oven to below 200 degrees $F$, then release pressure on the fixture and disassemble.

14. Place the bottom flange with the boundary sleeve, cement, and central loading tube still in place on the bench. Pour water on top of the cement insides the boundary sleeve, and apply 20 psi air to the bottom port. Observe the location of the bubbles to determine if the leak occurred at the outer pipe-cement interface, inner pipe-cement interface, or through a crack in the cement matrix. 


\section{(बSi) CSI Technologies}

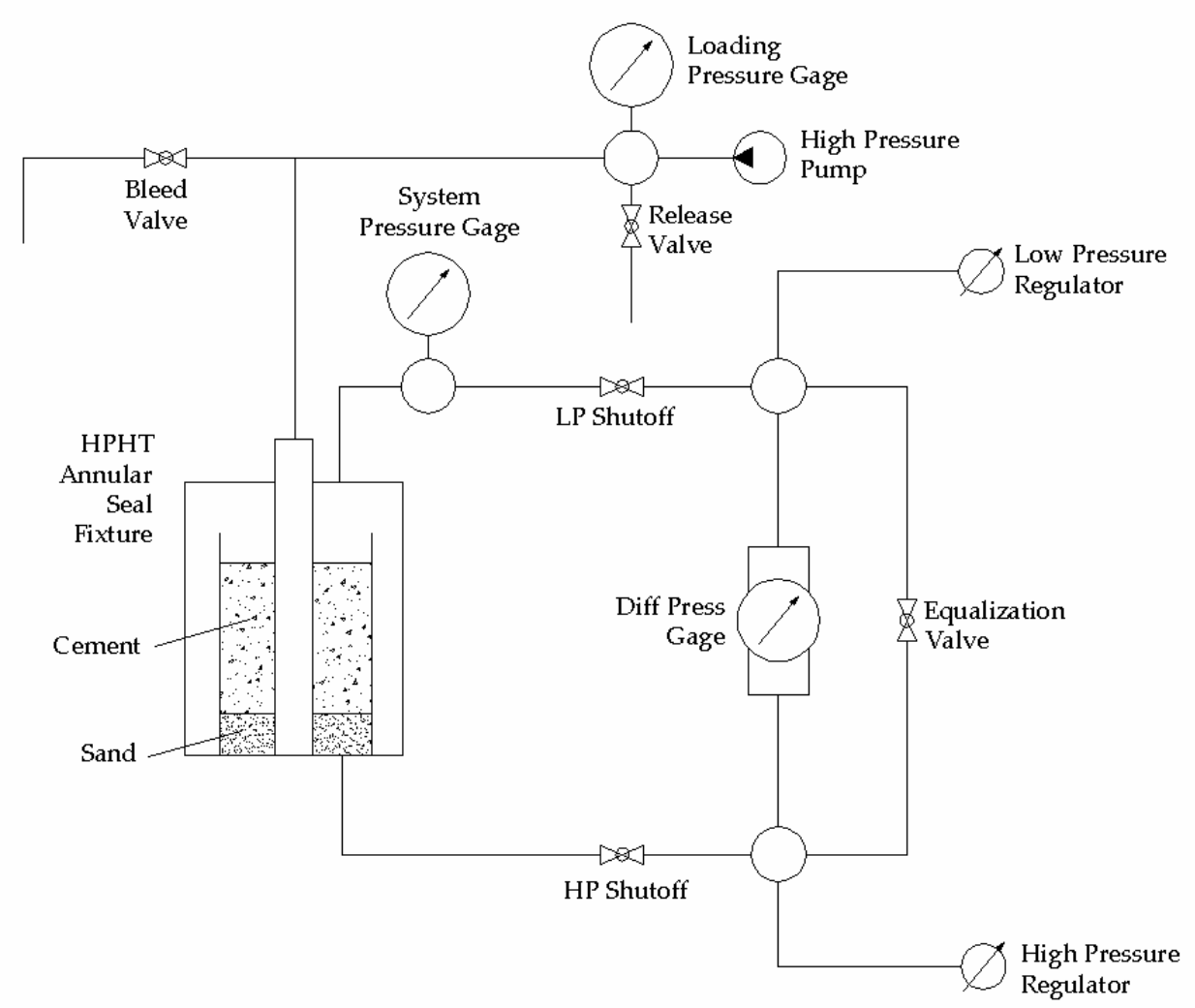

Figure 4-HPHT Annular Seal Test Schematic

Table 2 shows results of the Annular Seal Testing in hard formation. The numbers are the calculated energy required to fail the cement after curing, based on the number of cycles at each pressure and the internal volume of the loading tube. Results show that the Pre-Stressed Cement absorbs 5 times the energy before failure occurs than the Baseline cement system. Previous project publications listed a larger performance difference between Pre-Stressed cement and the baseline cement, but was due to a calculation error. 


\section{(BS) CSI Technologies}

Energy at each pressure level, in in-lbs.

\begin{tabular}{|r|c|c|c|c|c|c|}
\hline \multirow{2}{*}{ Pressure } & \multicolumn{3}{|c|}{ Baseline 99 } & \multicolumn{3}{c|}{ Pre-Stressed Cement } \\
\cline { 2 - 7 } & Test 1 & Test 2 & Test 3 & Test 1 & Test 2 & Test 3 \\
\hline 1,000 & 4,356 & 4,356 & 4,356 & 15,245 & 21,779 & 17,423 \\
2,000 & 8,712 & 8,712 & 8,712 & 30,491 & 43,558 & 34,847 \\
3,000 & 13,068 & 13,068 & 13,068 & 45,736 & 65,338 & 52,270 \\
4,000 & 17,423 & 17,423 & 17,423 & 60,982 & 87,117 & 69,693 \\
5,000 & 21,779 & 21,779 & 21,779 & 76,227 & 108,896 & 87,117 \\
6,000 & 26,135 & 26,135 & 26,135 & 91,473 & 130,675 & 104,540 \\
7,000 & 30,491 & 30,491 & 30,491 & 91,473 & 152,454 & 121,963 \\
8,000 & 34,847 & 17,423 & 17,423 & 104,540 & 174,234 & 139,387 \\
9,000 & 39,203 & 19,601 & 19,601 & 117,608 & 196,013 & 156,810 \\
10,000 & 21,779 & 21,779 & 21,779 & 130,675 & 217,792 & 152,454 \\
\hline Cum & 217,792 & 180,767 & 180,767 & 764,450 & $1,197,855$ & 936,505 \\
\cline { 2 - 7 } Average & \multicolumn{3}{|c|}{193,109} & \multicolumn{4}{c}{966,270} \\
\hline
\end{tabular}

Table 2 - Results of Hard Formation HPHT Annular Seal Testing

\section{Mechanical Shear Bond Testing}

After the cement sample fails in the HPHT Annular Seal testing, the central tube is pressed from the cement mass, and the force required to do so is recorded. The force is divided by the area of pipe in contact with the cement to calculate the mechanical shear bond. Traditionally, this shear bond is a measure of the strength of the chemical bond between the cement and the pipe. In the case of Pre-Stressed cement, there is also a mechanical component due to the contact stress between the cement and the steel caused by the cement expansion. Two types of tests have been used. The first pushed on the top of the central loading tube directly, but concerns of swelling the pipe due to the resistance of the cement make it possible to distort the values. Figure 5 shows the first version of the test setup schematically. The second version utilized a push rod inserted into the loading tube to push the pipe from the bottom (Figure 6). 


\section{(BS) CSI Technologies}

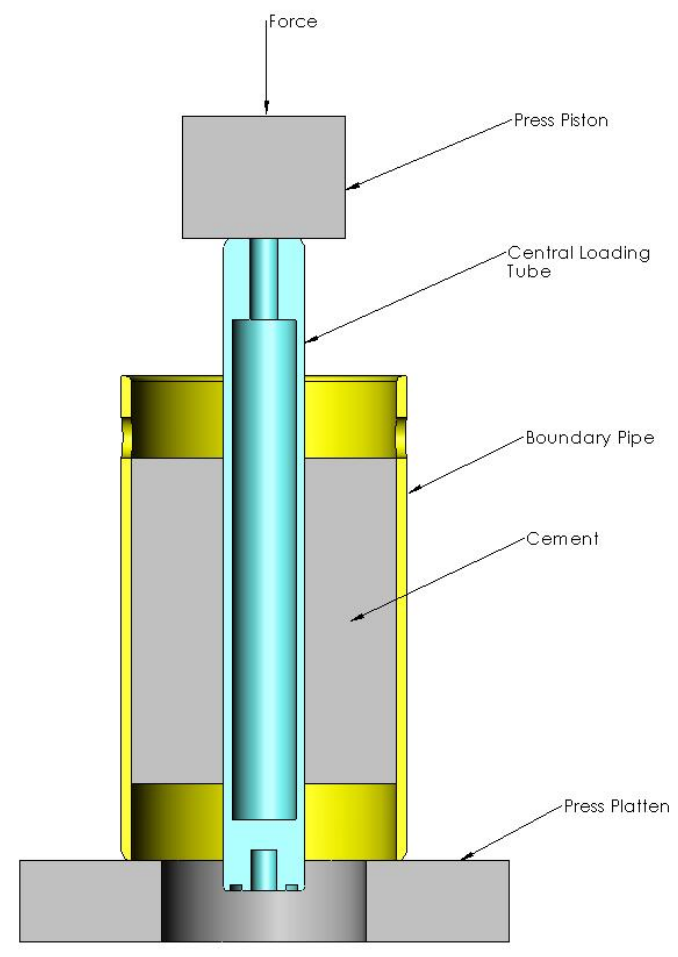

Figure 5 - Mechanical Shear Bond Test Setup - Push from Top

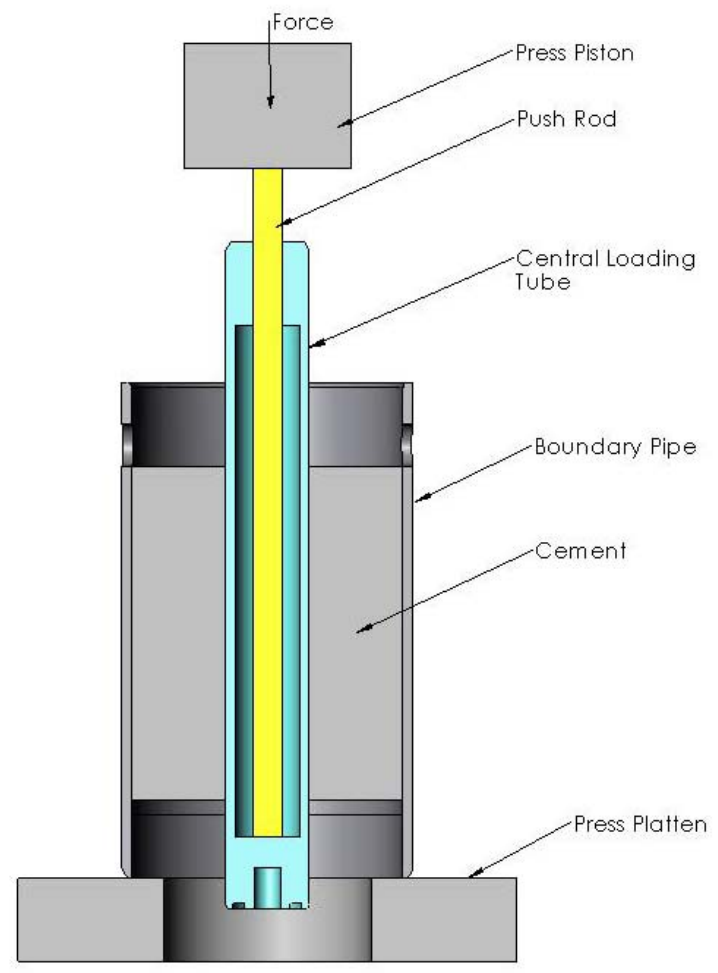

Figure 6-Mechanical Shear Bond Test Setup - Push from Bottom

Shear bond for Baseline 99 cement averaged approximately 140 psi. For the Pre-Stressed cement, the average shear bond over two samples was $1,840 \mathrm{psi}$, for an increase of approximately 13 times, utilizing the method illustrated in Figure 6. In the third sample of PreStressed cement, the weld between the loading tube and the end piece failed before the bond between the cement and the pipe was broken (Figure 7). 


\section{(8S) CSI Technologies}

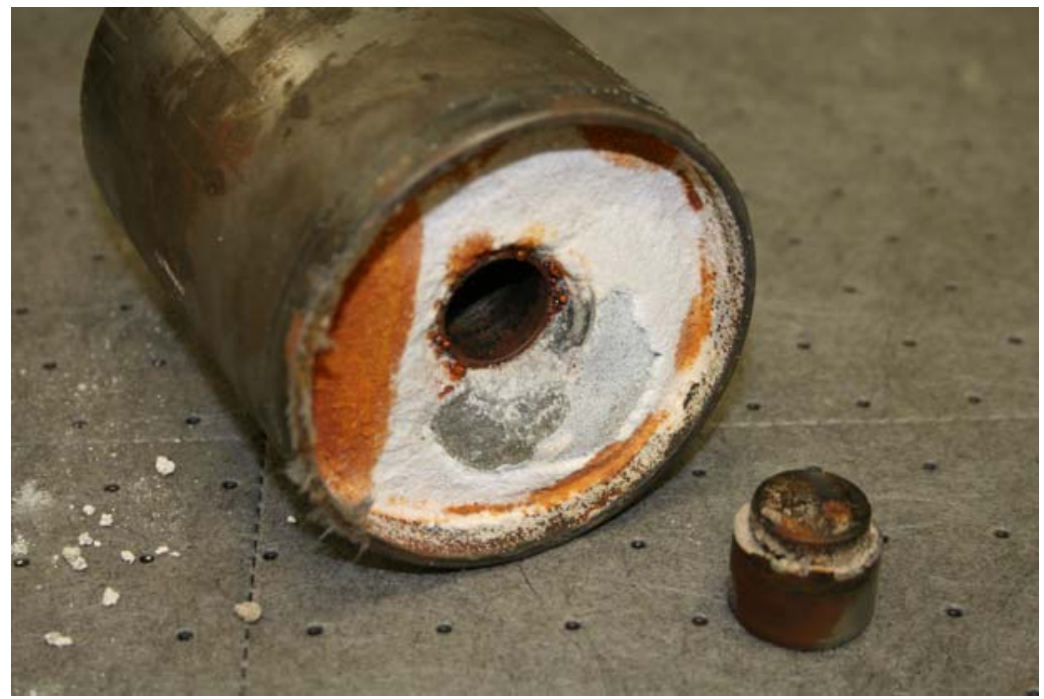

Figure 7 - Pre-Stressed cement - Pipe end weld broke before cement-pipe shear bond

In an earlier test on a Pre-Stressed cement that was not field-mixable utilizing the method shown in Figure 5, the application of force on the central loading tube bent the pipe without breaking the bond. The sleeve / cement / loading pipe assembly was then reversed so force could be applied to the "short end" of the loading tube. This effort buckled the loading pipe around the welded-in pipe end in a mushroom fashion, again without failing the bond. Figure 8 shows the resultant sectioned sample with the bent loading tube on the right and the mushroomed end on the left. 25,000 lbf was applied to the mushroomed end, supported by the 2-1/4" long cement plug. Without failing the bond, the cement supported 3,540 psi shear bond.

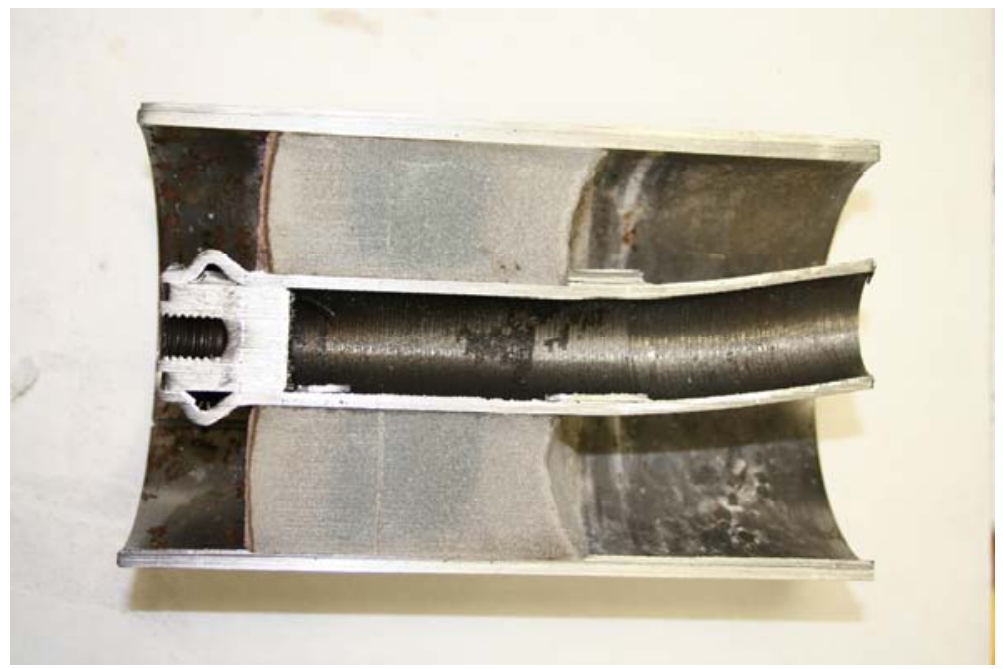

Figure 8 - Failed Shearbond Test 


\section{(BS) CSI Technologies}

\section{Hydraulic Bond Testing}

The resistance to various materials to allowing water to flow through or past a plug was measured at ambient temperatures. In this test, the cement or resin was cured at temperature and pressure in a pipe with either a sand bed or Ultra Spacer bottom so the sealant would not plug off the incoming water line. After curing, high-pressure water was pumped in until the bond broke, allowing water to flow from the open end of the pipe. Table 3 summarizes the results of the hydraulic bond testing, and Figure 9 shows the test setup schematically.

\begin{tabular}{|l|l|}
\hline Sealant Material & Hydraulic Bond - psi \\
\hline Baseline 99 Cement & 3,800 \\
\hline Pre-Stressed Cement & 6,000 \\
\hline Resin & 6,425 \\
\hline
\end{tabular}

Table 3 - Hydraulic Bond Test Results

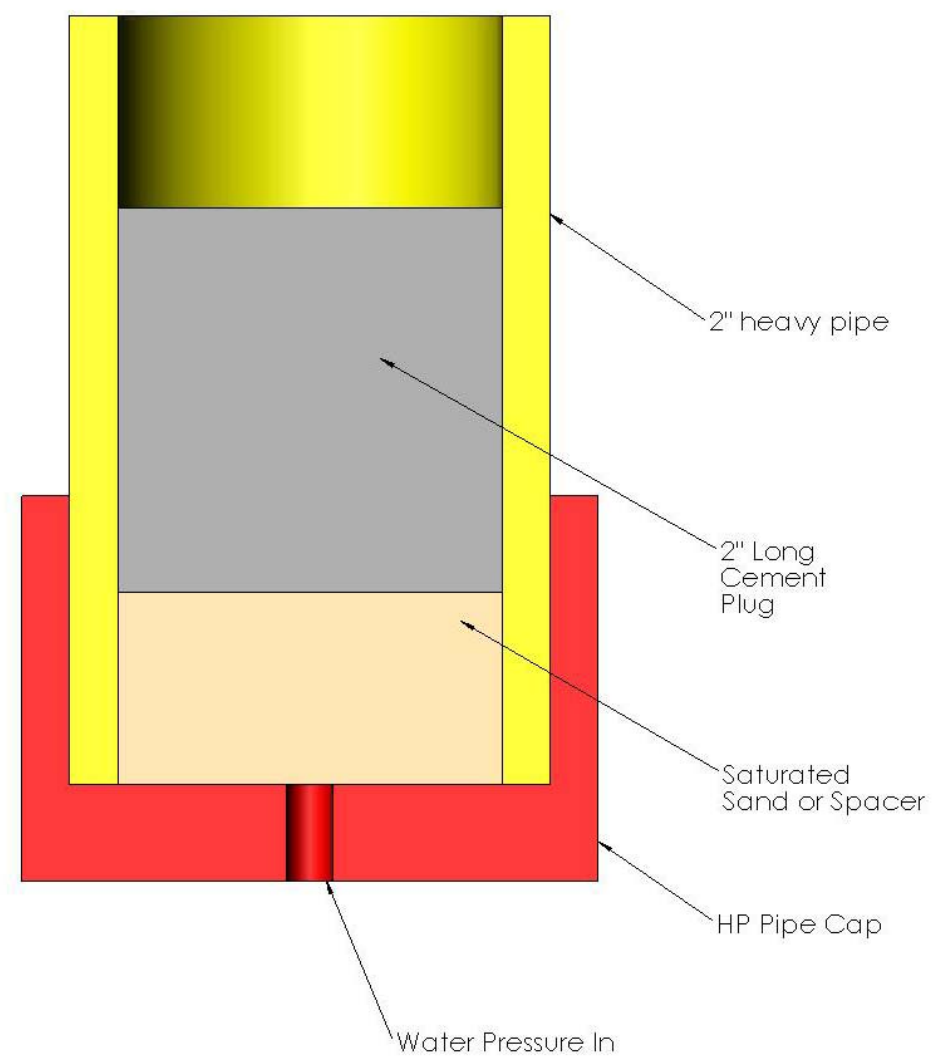

Figure 9 - Hydraulic Bond Test Setup 


\section{CSI Technologies}

\section{HPHT Expansion Testing}

Pre-Stressed cement achieves superior performance in the lab when compared with conventional HPHT cements by exhibiting significant expansive characteristics during and after curing. This behavior augments the performance by creating a mechanical pressure component to the traditional chemical bonding of Portland cement to pipe. Efforts to measure the expansion in real time as the cement cures has so far eluded CSI researchers, due to limitations in available force measuring devices (see Task 5 discussion). Other methods show some promise, but equipment rebuild issues have not allowed successful real time tests to be completed to date. Work is continuing on this issue.

However, success has been achieved by measuring the dimensional change in the HPHT Annular Seal boundary sleeves (and in the hard formation case, the central loading tube) after those tests are complete. While these measurements give only a gross expansion value rather than a continuous measurement during and after curing, it does allow comparisons with confinement integrity, allowing an additional performance predictive tool when applying the material to real wellbores. FEA studies were conducted to determine the internal pressures required for the various sleeve materials to produce the observed diametral change from the test specimens. As expected, it was found that higher levels of confinement resulted in higher internal pressures generated by the cement expansion. Table 1, contained earlier in this report under the discussion of Expansion Theory, contains the expansion pressures produced in actual samples.

\section{Pre-Stressed Cement HPHT Mud Channel Testing}

Concerns were expressed by some members of the DeepTrek Advisory Committee regarding the viability of the Pre-Stressed cement mass when confinement was less than $100 \%$ effective. To simulate that situation, a test apparatus was constructed to simulate a mud channel on top of cement in a horizontal well in a geometrically- and scale-correct arrangement. The pipe-in-pipe apparatus was designed with 5.5" $\times 5.0^{\prime \prime}$ inner casing and 8-5/8" x 7-3/8" outer casing, partially filled with Pre-Stressed Cement with a channel above the cement of approximately $.75^{\prime \prime}$. Water was used to fill the pipe (in the mud channel area), and connected to a pressure regulator set at 200 psi. Two fixtures were built, to simulate both water-wet and oil-wet conditions. In the oilwet case, three quarts of 30 weight motor oil was poured in the annulus and rolled to thoroughly oil-wet all internal surfaces prior to the addition of the cement. After the internal steel surfaces were thoroughly oil-wet, the oil was drained from the fixture and the cement was poured in. The point of this exercise was to determine if the cement would bond to the oil-wet surfaces. The fixtures were placed in a $300 \mathrm{deg} F$ oven and allowed to cure for 48 hours, at which point they were removed and sectioned to observe the cement in the annulus. The results of the test were a thin layer of friable cement on top of the cement mass, but the overwhelming majority of the cement was hard, dense, and competent. No differences in the cement mass were found between the water-wet and oil-wet cases. Figures 10 and 11 show the cut section after the test was complete. The friable portion of the cement is easily visible as darker gray and crumbly, while the competent cement is lighter gray in color. This test proved that Pre-Stressed cement will function effectively even in partial confinement conditions. 


\section{(BS) CSI Technologies}

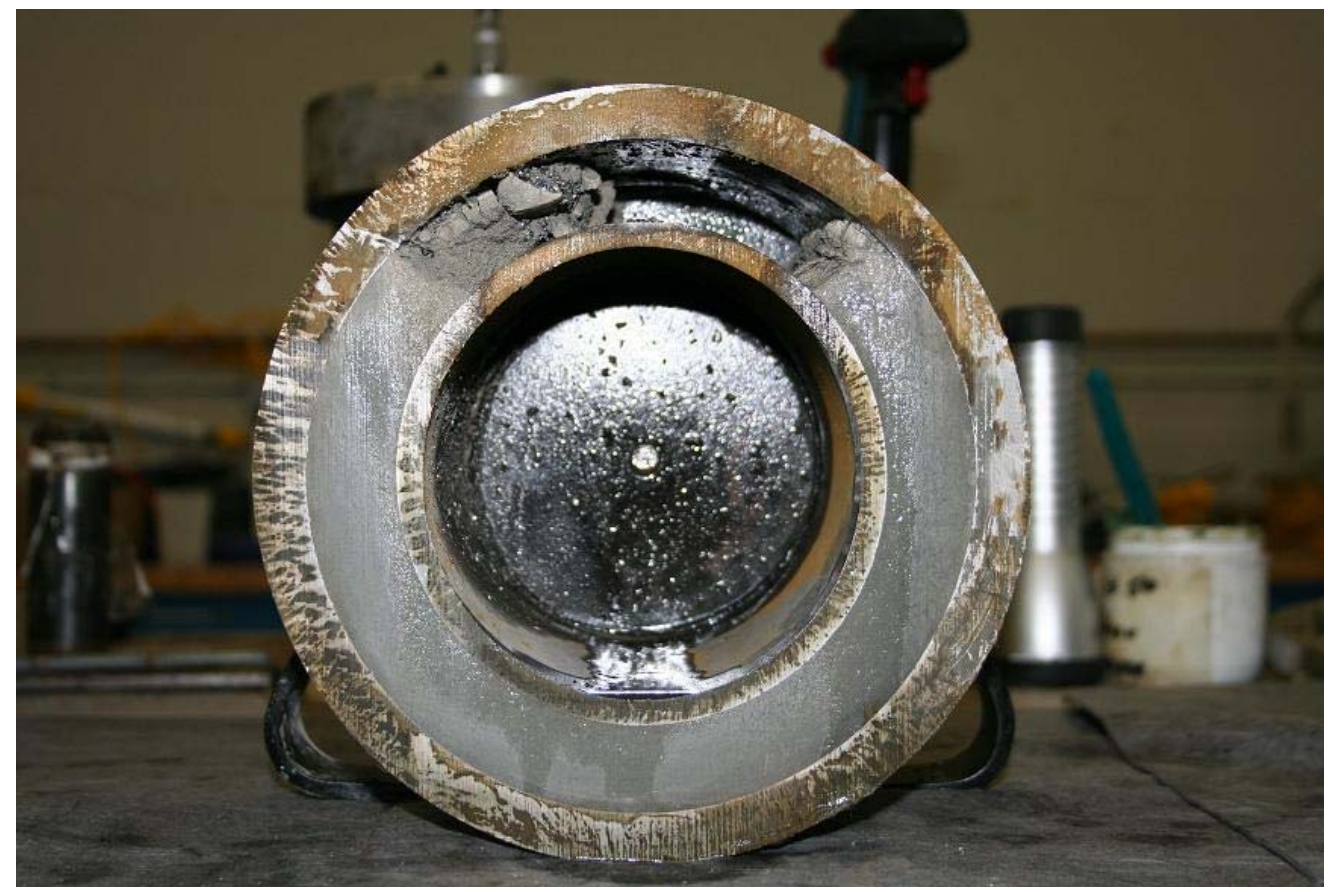

Figure 10 - Cut section of Mud Channel Simulation Test Apparatus

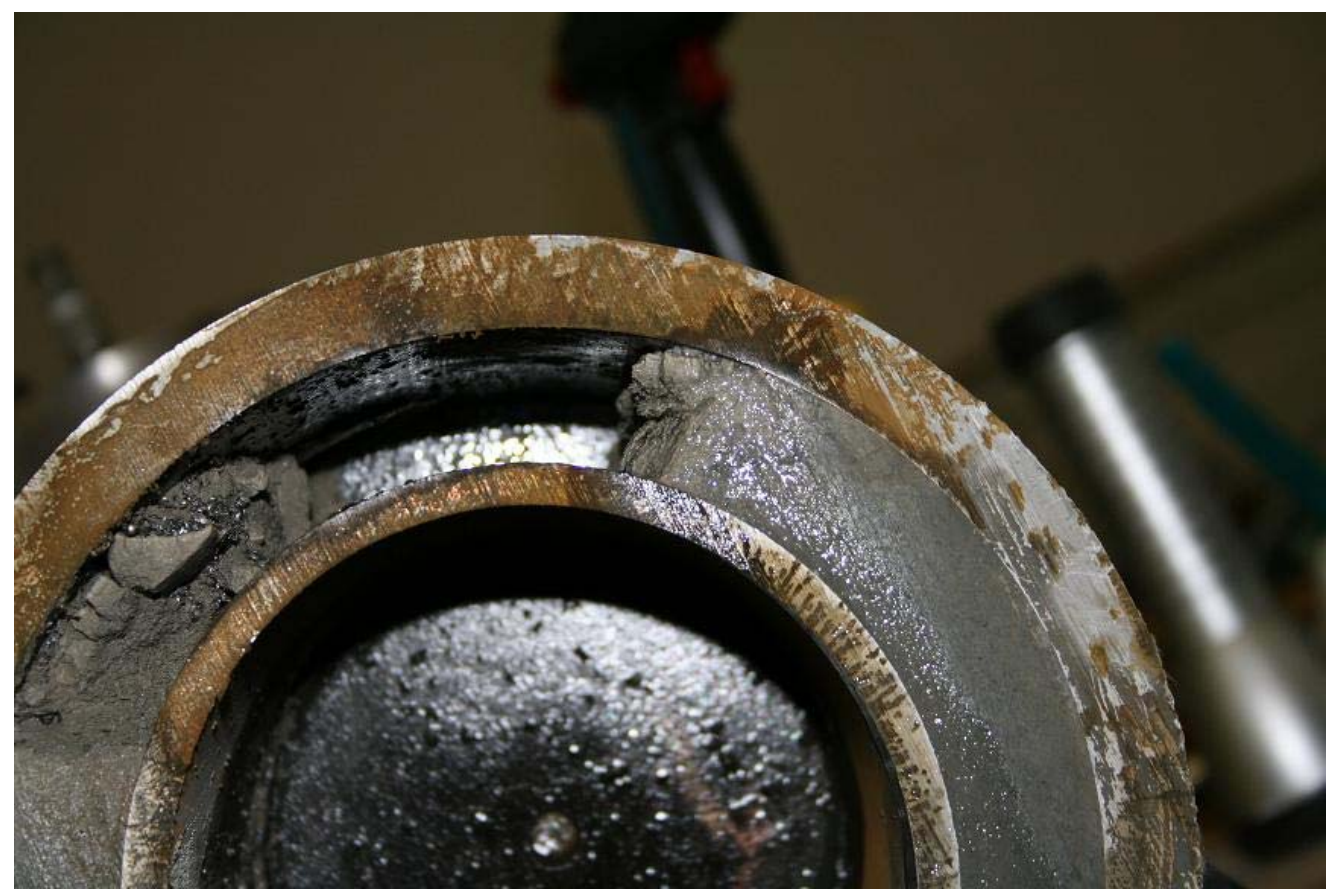

Figure 11 - Closeup - Cut section of Mud Channel Simulation Test Apparatus 


\section{(BS) CSI Technologies}

Task 3 - Evaluate the product's performance in large-scale mixing, shearing, and drillout studies.

\section{Epoxy Resin}

Resin was mixed in large scale yard test on April 7, 2005. A conventional batch blender equipped with a square mixing tank, agitator, and diaphragm transfer pump was rented and set up at the CSI facilities. Resin was manually poured into the mixing tank, after which a premeasured amount of activator was added.The mixture was mixed and held in the tank for the desired amount of time, and then pumped into 55-gallon drums. Samples were taken for subsequent analysis. For this test, Figures 12 through 14 show various stages of the process for this pilot test.

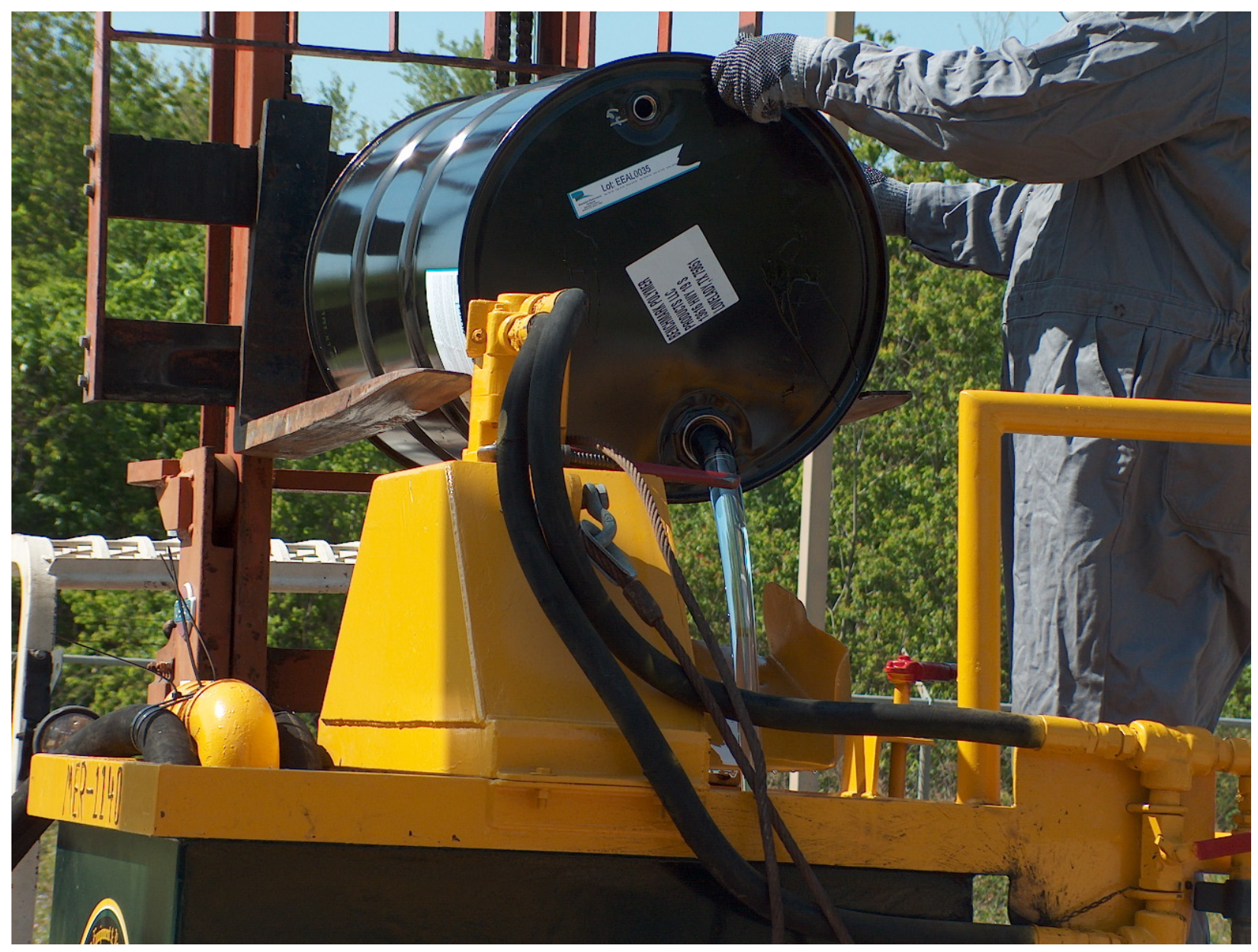

Figure 12 - Manually Introducing Resin into the Mixing Tank 


\section{(48) CSI Technologies}

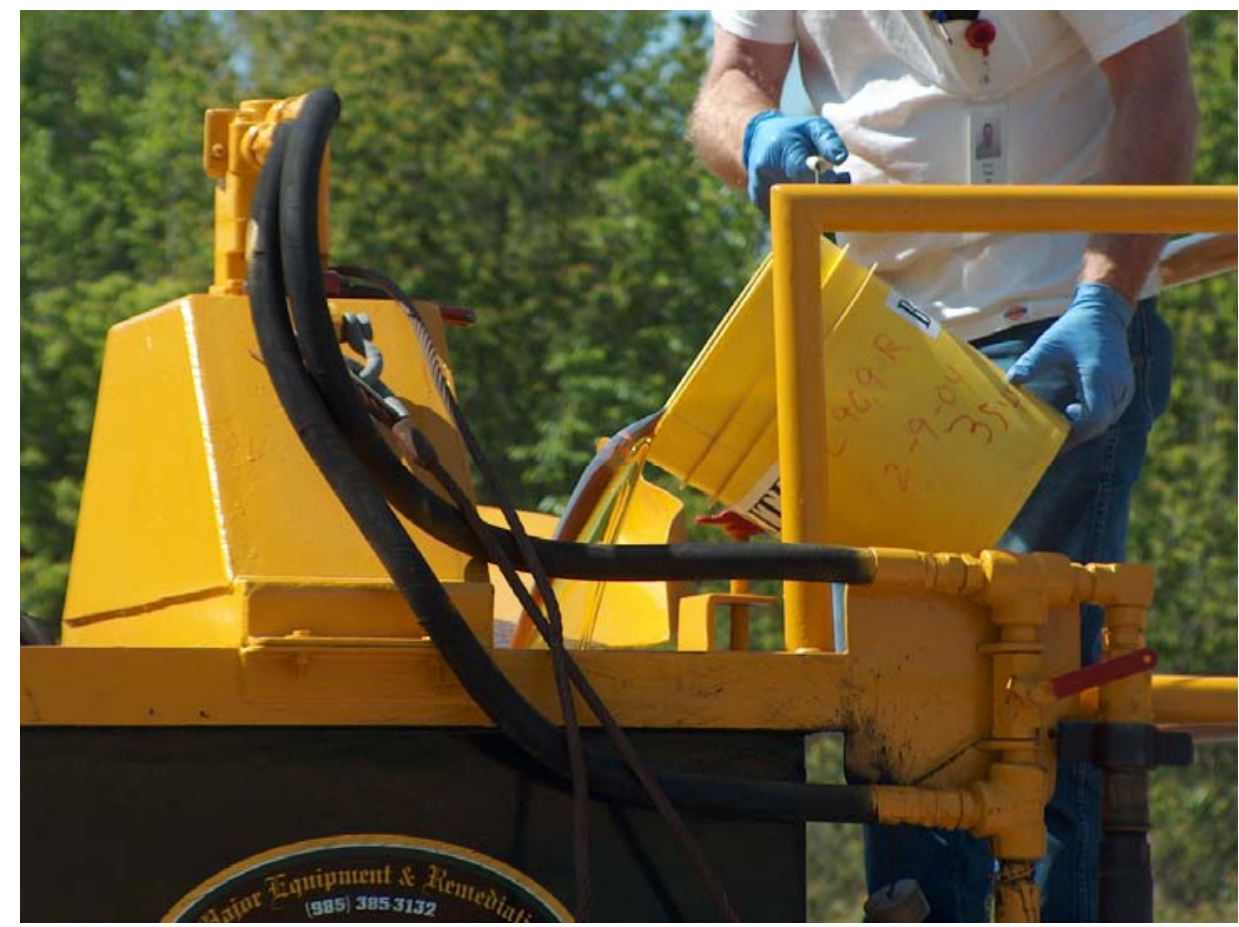

Figure 13 - Manually Introducing Activator into the Mixing Tank

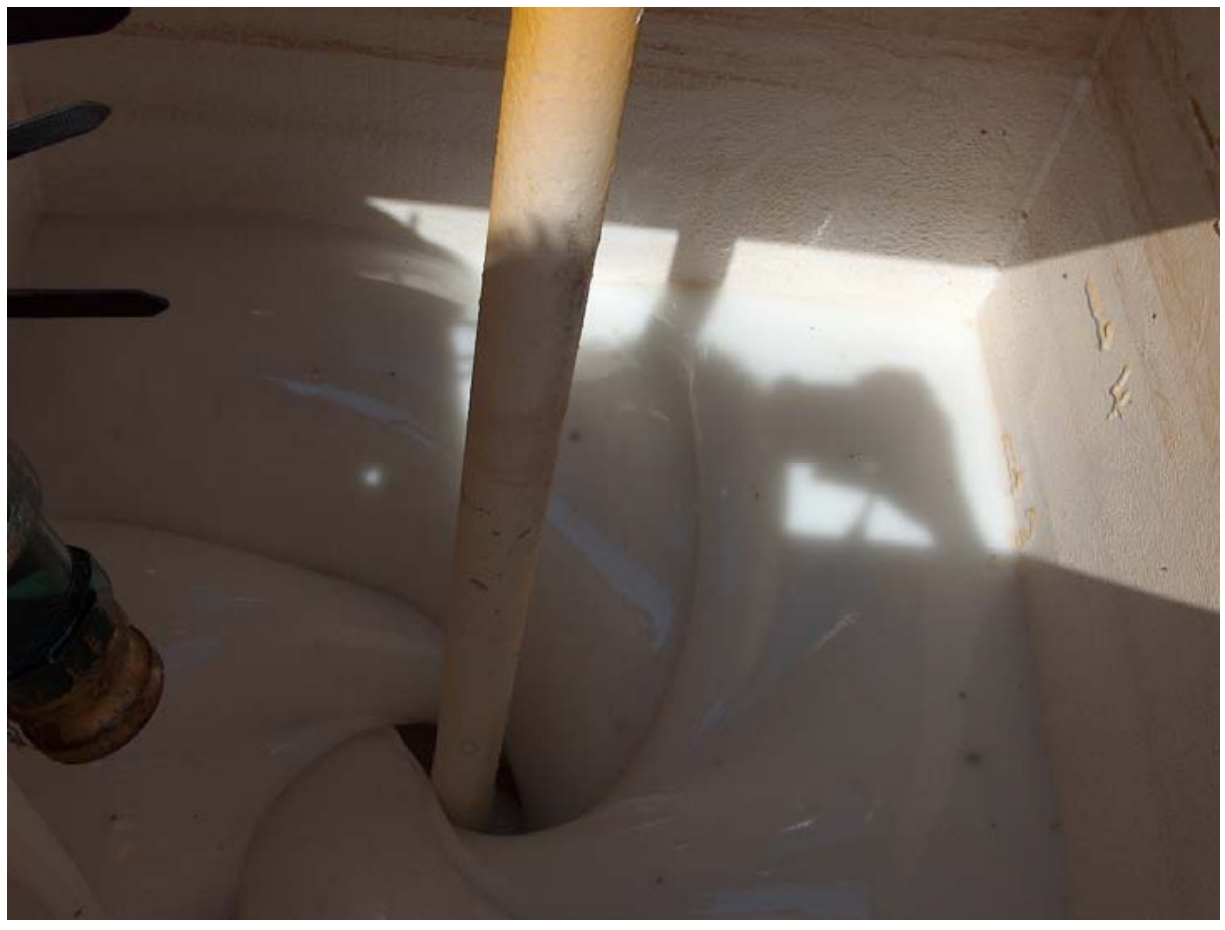

Figure 14 - Resin / Activator Mixture in the Mixing Tank 


\section{(영 CSI Technologies}

The epoxy resin product requires significantly different handling, mixing, and cleanup procedures than Portland cement:

\section{Handling:}

Personnel must be protected from the fumes from the resin and the activator, and the product produces skin irritation after contact. For this reason, steps must be taken to protect personnel from breathing resin vapors and contact with the product itself. Figure 15 shows typical Personal Protection Equipment recommended for the handling of resin: respirator, gloves, coveralls, and safety glasses.

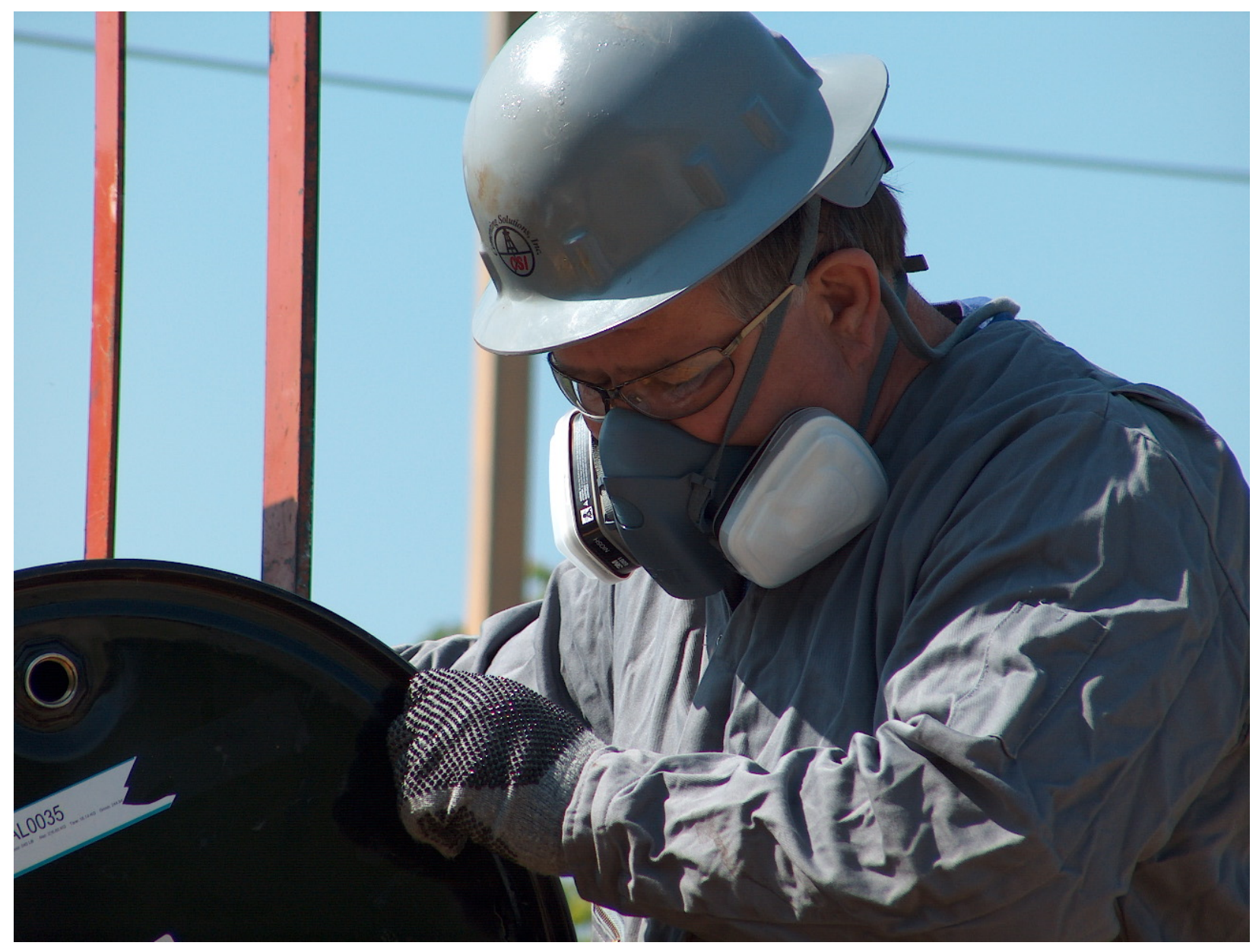

Figure 15 - PPE for Resin Handling

\section{Mixing:}

The material is extremely exothermic when the reaction begins. Temperatures in excess of 400 degrees have been measured while the material is setting. For this reason, care must be taken to assure that the material does not set in the surface mixing and pumping equipment. Not only can the material damage the equipment, but the potential for initiating fire is present if allowed to attain these temperatures on the surface. Additionally, unpressurized water in contact with 


\section{(BS) CSI Technologies}

or incorporated into the resin during setting can flash to steam. Care must be taken to assure that any residual water contained in the mixing equipment or transfer lines is removed prior to mixing the product. In the wellbore, enough pressure will exist or can be applied to the material to prevent vaporization of water in contact with the resin. During the test, an attempt was made to remove heat during the exotherm sufficient to prevent boiling, but figure 16 shows the futility of the effort. Resin was placed in 5-gallon buckets, which were then placed in a holding tank full of ice. In this photo, incorporated trace water from the mixing system and thermal expansion of the material caused the material to overflow the buckets. Temperature measurements of the product during this process indicated that the internal temperature rise was not affected by the attempt to cool the product. This exercise underscored the importance of removing water contamination and designing the product so that a safe surface handling time is achieved.

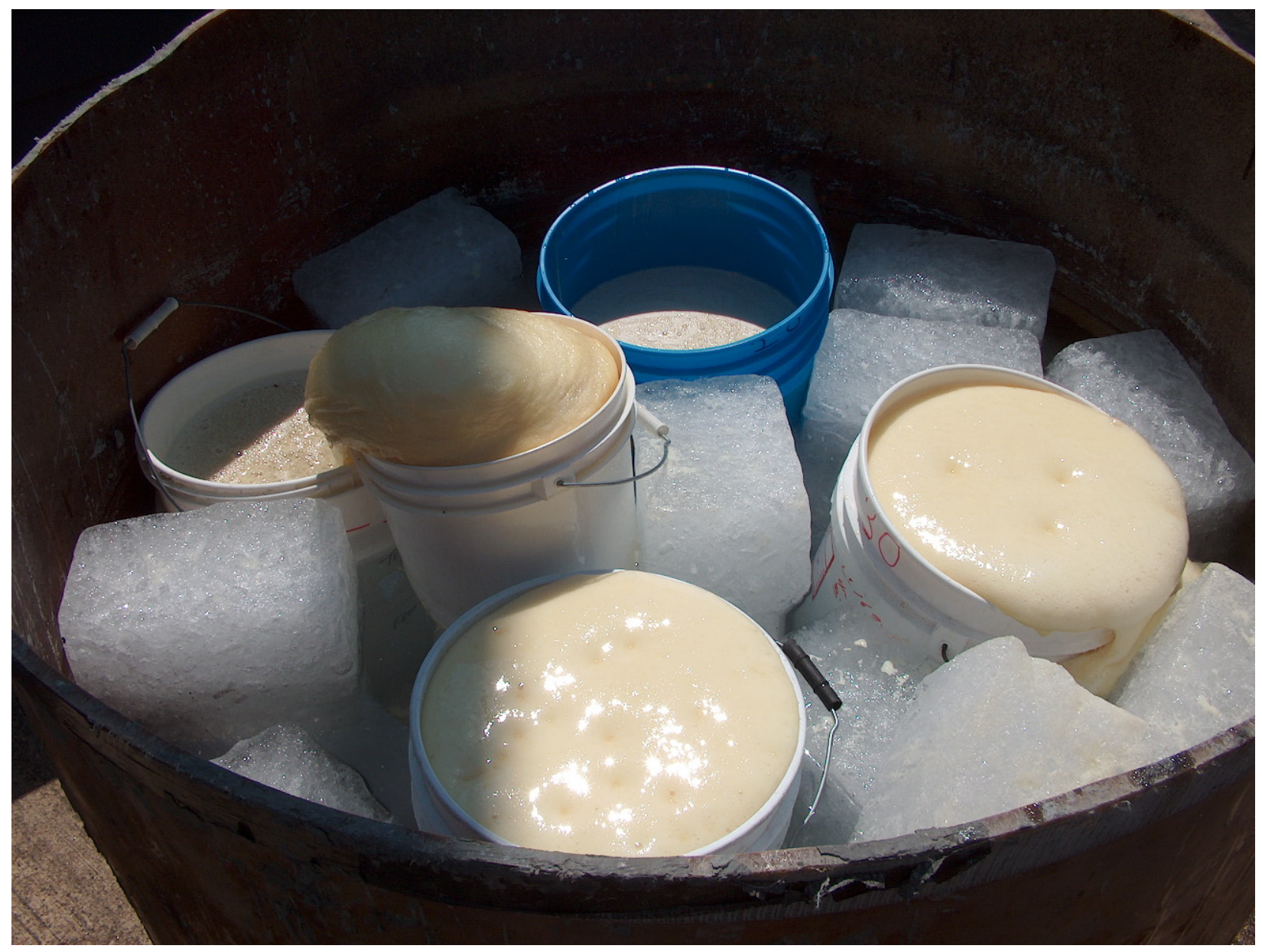

Figure 16 - Heat of Activation

\section{Cleanup:}

Resin does not mix with water, offering potentially advantageous placement scenarios in wellbores. However, this also creates difficulties with cleanup of handling, mixing, and 


\section{(BS) CSI Technologies}

pumping equipment. A mixture of methanol and water is effective in dissolving the unset resin and cleaning surface equipment. Cleanup therefore requires a volume of methanol on location, the ability to introduce the methanol / water mixture into the equipment, and subsequent storage and disposal of the fluid after cleanup is complete.

While the issues noted are significantly different than the handling of conventional cement they are manageable with conventional mixing and pumping equipment in the oilfield today. To date, field tests have been conducted with equipment similar to the equipment shown in the year test because of the small volumes of the treatments. In practice, it is possible to build a closed continuous mixing system utilizing controlled resin and activator injection rates, while protecting the operators from exposure to the material. Preliminary designs have been completed for a time when they are required.

The resin product is a liquid - liquid system that is not shear-sensitive, so no significant effort was made to shear the material in a flow loop. A sample was poured and cured for drillout studies. A 1.75" diameter carbide coiled tubing milling bit was obtained and taken to a machine shop. The bit was situated in the lathe dead center, and the cured resin was placed in the faceplate. The lathe was turned at a very slow speed (100 rpm), consistent with drilling operations, and the milling bit was advanced manually approximately 3.5 " into the resin. The material cut cleanly without gumming or fouling the bit. Cuttings were competent and can easily be removed from the wellbore with conventional circulation procedures. Figures 17 and 18 show the resultant drilled sample. Subsequent field tests with the resin material have been drilled with no problems.

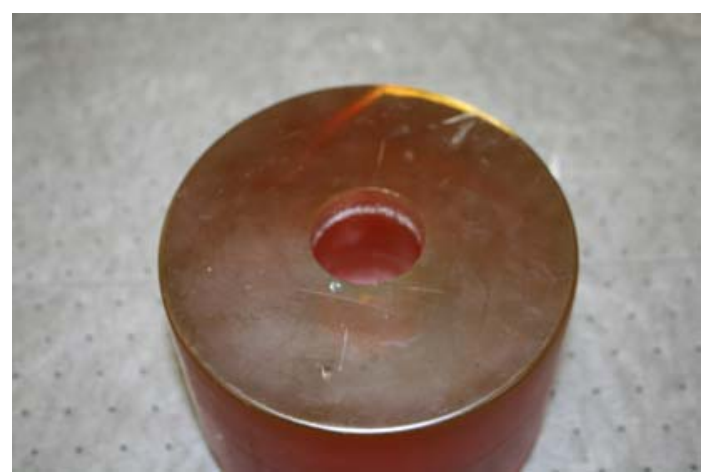

Figure 17 - Milled Resin Sample

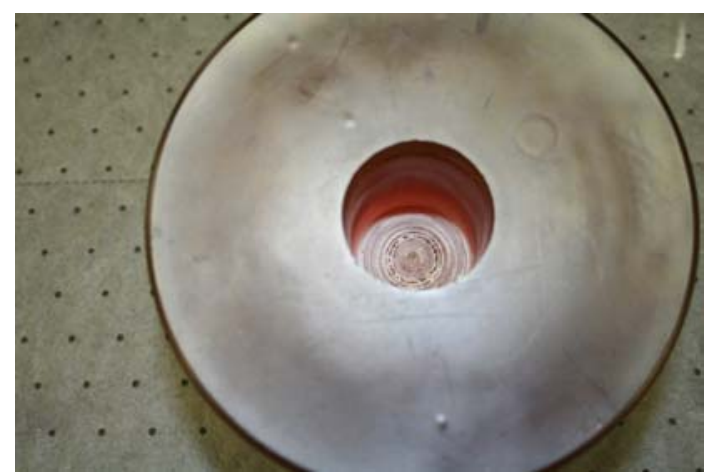

Figure 18 - Milled Resin Sample 


\section{(8.8) CSI Technologies}

\section{Pre-Stressed Cement}

Pre-Stressed Cement required no significant work in mixing, shearing, and drillout due to it's legacy as Portland cement with unconventional proportions of conventional additives. The primary challenge with this material is to retard it sufficiently for HPHT applications. To date, the material has been successfully retarded to $300 \mathrm{deg}$ F static, and work is continuing to control the material at higher temperatures with different organic acids, phosphates, and reformulations of the basic recipe. Appendix B contains sample thickening time and rheology test reports for Pre-Stressed cement.

\section{Task 4 - Test the composion's performance in a research test well}

\section{Epoxy Resin}

Over 50 jobs were performed in test wells, generally in order of ascending difficulty in terms of HPHT conditions. The first jobs were simple shallow, low temperature P\&A jobs, performed to allow the operator to abandon wells in the Gulf. Eventually, squeeze jobs were performed up to 250 deg F with success.

Two jobs were chosen as Research Test Wells for the purpose of this project. The first was a perforation squeeze job conducted in November of 2005 for Chevron in Bay Marchand, LA. Depth was approximately 7,500 feet and the temperature approximately $150 \mathrm{deg}$ F. Fluids utilized included a staged placement of $4 \mathrm{bbl}$ Ultra Spacer and $4 \mathrm{bbl}$ Resin. The spacer was used to temporarily seal the perforations so the resin would not be lost through to the formation, allowing the resin to remain in the casing. The fluids were bullheaded down tubing and successfully sealed the perforations, as verified by post-job pressure test.

The second job was conducted for Stone Energy in March, 2006, and was a P\&A job with perforations at 10,200' depth and $250 \mathrm{deg}$ F. The well was in an inland waterway near Lafitte, LA. The job was performed to remediate an unsuccessful conventional cement plug. The injection rate was established at $2 \mathrm{bpm}$ at 2,000 psi, while holding backpressure on the casing to prevent the well from kicking. $5 \mathrm{bbl}$ of resin, barite-weighted to $16.5 \mathrm{lb} /$ gal were pumped, followed by $40 \mathrm{bbl}$ of displacement fluid. After displacement, the well was shut in at 2,100 psi for 14 hours. Post-job pressure testing was successful, and the job was ended.

Complete job reports are included in Appendix C.

\section{Pre-Stressed Cement}

To date, no jobs have been completed with the Pre-Stressed cement, but CSI is working with Anadarko Petroleum to initiate field testing in 2006. Due to the more "conventional" nature of the Portland Cement material, there are no handling, mixing, or pumping procedure changes required to apply the material in the field. Further, extensive laboratory testing is required to overcome misconceptions regarding the controllability and the benefits of expanding cement. Expanding cement has been studied in the industry in the past, but the belief that it cannot be retarded at high temperatures and applications in unconsolidated formations have masked it's positive benefits in more competent zones. 


\section{(48) CSI Technologies}

\section{Task 5 - Phase for-cost extension - Develop HPHT Annular Seal, Direct Tensile, and HPHT}

Expansion Tests

\section{Introduction}

As this project progressed, it became obvious that more specialized laboratory tests were needed to adequately test sealant materials in the lab. These tests needed to be conducted at temperature and pressure in real time. While measuring various mechanical properties of sealants is instructive, no combination of properties has to date adequately predicted performance in the wellbore environment. The Annular Seal test developed at CSI was an attempt to directly measure performance, but was done at ambient temperature and pressure. Extending the testing principle to high temperatures and pressures required significant equipment design and development as well as protocol development. To measure properties, a direct tensile test was conceived that would enable measurement of tensile strength, tensile fatigue, and tensile Young's Modulus using conventional compressive test machines. Finally, measuring expansion at temperature and pressure in real time would help answer the question if Pre-Stressed cement functioned by expanding during the curing process or post-set. This information is useful in the design of the slurry, so the expansion is controllable.

\section{HPHT Annular Seal}

Figure 19 shows the HPHT Annular Seal apparatus in cross section.

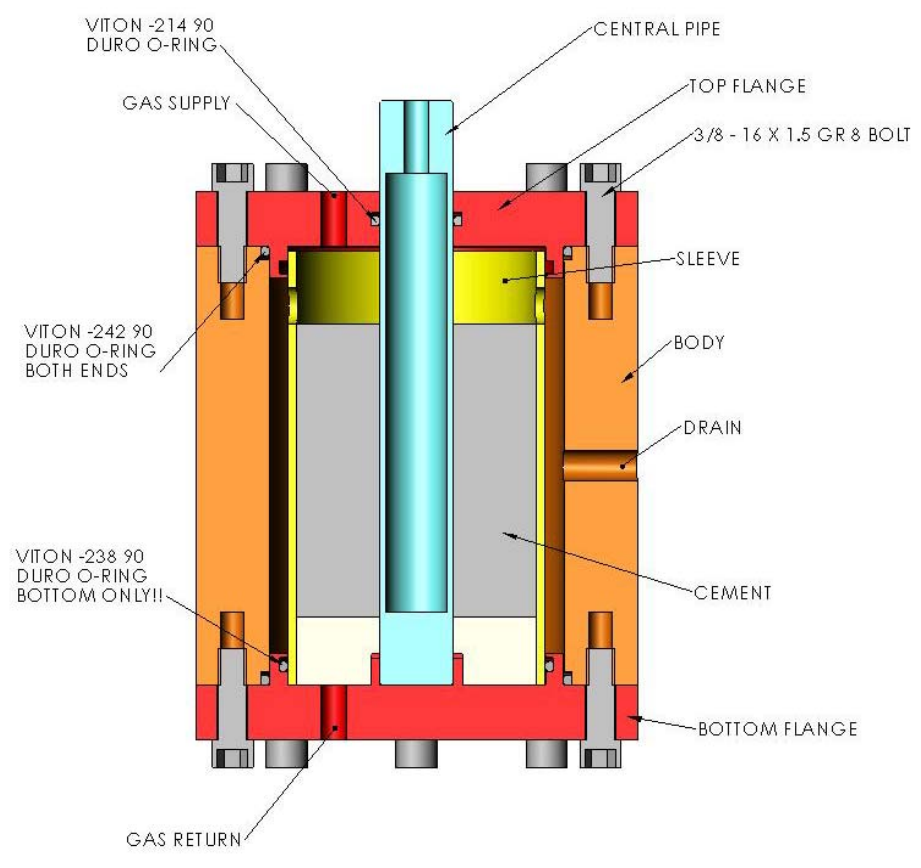

Figure 19 - HPHT Annular Seal Apparatus 


\section{CSI Technologies}

The body and top and bottom flanges are designed to safely withstand $300 \mathrm{deg} F$ temperatures and 3,000 psi internal pressure. The tests were run at 1,500 psi due to limitations on the flexible hoses used to transmit fluid and pressures inside the oven, and potential limits of the o-rings used to maintain internal pressure. The cement sets in the annulus between the boundary sleeve and the central loading pipe. Between the cement and the bottom flange is a layer of saturated sand, so the cement does not plug the gas inlet passage. Water is placed in the annulus between the boundary sleeve and the fixture body, and also on top of the cement up to the top flange. The boundary sleeve contains cross-drilled holes near the top to assure that the cement has all the water required for the hydration reaction to be completed successfully. The test is conducted according to the testing protocol listed earlier in this report.

The boundary sleeves utilized in the HPHT Annular Seal test were designed to simulate the behavior of various formation types, essentially with an infinite radius from the wellbore, with finite wall thickness tubes of various dimensions. To simulate an infinite formation with a specific Young's Modulus, it is possible to use materials of higher Young's Modulus in thinner sections. The materials were chosen to maintain their properties at elevated temperatures experienced during the test. After extensive Finite Element Analysis (FEA) modeling, the strong formation (Young's Modulus of 5 million psi) was simulated with a steel sleeve 3.5" OD and 3.26 ID. In order to maintain comparisons, other sleeves were required to have the same ID as the steel sleeve. Aluminum has the lowest Young's Modulus (approx 11 million) of all readilyavailable metals, and simulations showed that utilizing a wall thickness of $0.05^{\prime \prime}$ gave a reasonable approximation of an infinite formation of 1 million psi Young's Modulus, in terms of displacement at the outer cement-formation interface. Similarly, PEEK (an engineering plastic with Young's Modulus of approx 1.8 million psi) was utilized to simulate the weak formation of approximately 160,000 psi Young's Modulus.

\section{Direct Tensile Test}

The direct tensile test fixture was designed to allow direct measurement of tensile strength (as opposed to the indirect Brazilian method commonly employed in oilfield laboratories). The intent of the test was to allow Pre-Stressed cement to be tested after curing in confined conditions. Advantages to this apparatus is the ability to cure in a confined condition, measurement of tensile strength, tensile fatigue, and tensile Young's Modulus. Figure 20 shows the test cylinders in cross section.

The two halves are bolted together, and the end plug is inserted and bottom half. The figure shows an end plus installed in the top half as well, which is used only for Pre-Stressed cement. The green cylinder to the right of the fixture is an LVDT, used to measure displacement during the test for the purpose of calculating Young's Modulus.

After the cement is cured, the bolts holding the two halves together are removed, and the cylinder assembly is installed in a tensile frame (Figure 21). The tensile frame is designed to produce tensile force (pulling the two cylinders apart) when placed in a compression test machine. The LVDT is installed to measure displacement. 


\section{(BS) CSI Technologies}

In operation, the cement fractures at the interface between the two cylinder halves.

Displacement measured by the LVDT is compared with the distance between the two internal lands to calculate strain.

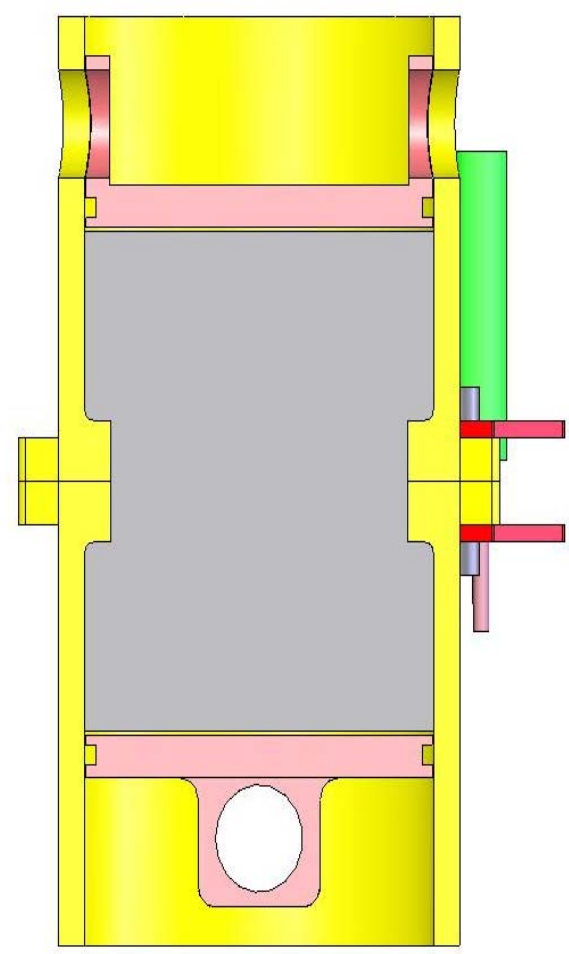

Figure 20 - Direct Tensile Test Fixture

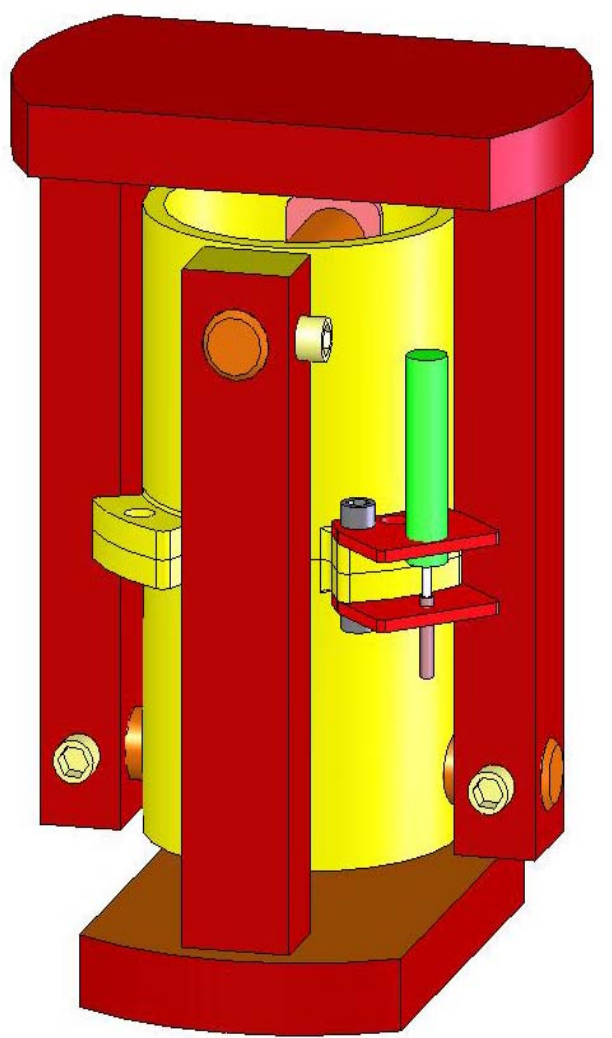

Figure 21 - Direct Tensile Test Assembly 


\section{(8SI) CSI Technologies}

In practice, the system has generated disappointing results when utilized with both Resin as well as Pre-Stressed cement. In the case of resin, the material's rubber-like consistency in the unconfined loading scenario causes the material to tear easily at the interface between the cylinders. Resin tensile strength is very difficult to measure by whatever method is utilized. In the Brazilian method, the material deformed dramatically without fracturing until a load was employed for calculations at the point the material began to fragment (although a classic vertical split was not present). In the case of Pre-Stressed cement, the lack of confinement in the load direction makes the material break easily at the interface. Ironically, the test has worked extremely well for conventional non-expanding cements, for which the test has been utilized for tensile fatigue and the calculation of tensile Young's Modulus.

\section{HPHT Expansion}

This test was intended to measure the expansive characteristics of Pre-Stressed cement during and after the curing period. In the original design, the cement was cured inside split sleeves that were placed in a pressure vessel, which was placed in an oven. Strain gage force transducers were to be utilized to measure the force generated by the expanding material. Although the force transducers were rated for use at $300 \mathrm{deg} F$, the response of the devices at temperature was not predictable, repeatable, nor stable. The devices continued to change with no applied force for up to 100 hours into a no-load calibration test, and repeated calibration tests revealed that the signal from the same device changed at temperature from test to test. After repeated attempts to calibrate the devices, the concept was abandoned.

The next attempt to continuously measure expansion centered on the pressure increase generated as the expanding cement squeezed the central loading tube in the HPHT Annular Seal apparatus. For this purpose, the central loading tube was machined down to 0.040 " wall thickness to maximize sensitivity. Tests with a fluid-filled central tube resulted in very large pressure increases during the heating period, masking the relatively small pressure rise during the expansion. This resulted in an inability to find a pressure transducer with sufficient range and precision to accurately measure the small volume change due to expansion. Subsequent tests with nitrogen in the loading tube resulted in a manageable pressure rise, but difficulty in sealing the apparatus sufficiently.

Current efforts seek to maintain the thinwall central tube at a constant pressure with a Ruska pump during the heatup, curing, and postcure periods and accurately measuring the fluid withdrawn from the tube to measure volume reduction due to expansion. In the calibration attempts, the pump obtained for the service would not hold pressure due to packing leaks, so the pump will be rebuilt. Efforts will continue after the pump is returned. 


\section{(BS) CSI Technologies}

\section{Appendix A - Resin Design Matrix and Thickening Time Tests}

80 Deg F Table

Percentage of Component to base Resin

\begin{tabular}{|c|c|c|c|c|c|c|}
\hline Comp A & Comp B & Comp C & Comp D & Comp E & $\begin{array}{c}\text { Unpump } \\
\text { Time }\end{array}$ & 24 Hr Set \\
\hline 100 & & & & & $1: 24$ & hard \\
\hline 65 & & & & & $1: 22$ & hard \\
\hline 35 & & & & & $2: 16$ & hard \\
\hline 25 & & & & & $3: 12$ & hard \\
\hline 24 & & & & & $4: 00$ & hard \\
\hline 23 & & & & & $5: 00$ & hard \\
\hline 20 & & & & & $5: 40$ & soft \\
\hline 15 & & & & & $6: 00$ & soft \\
\hline 10 & & & & & $7: 00$ & no set \\
\hline 5 & & 10 & & & $5: 00$ & no set \\
\hline 25 & & 10 & & & $5: 00$ & soft \\
\hline 30 & & 10 & & & $5: 00$ & hard rubber \\
\hline 35 & & 10 & & & $5: 00$ & hard rubber \\
\hline 40 & & 10 & & & $5: 00$ & hard rubber \\
\hline 45 & & 20 & & & $6: 00$ & soft \\
\hline 25 & & 20 & & & $6: 20$ & soft \\
\hline 30 & & 20 & & & $5: 20$ & soft \\
\hline 35 & & 20 & & & $5: 20$ & hard rubber \\
\hline 40 & & & & & & \\
\hline 45 & & & & & & \\
\hline
\end{tabular}

100 Deg F Table - Percentage of Component to base Resin

\begin{tabular}{|c|c|c|c|c|c|c|}
\hline Comp A & Comp B & Comp C & Comp D & Comp E & $\begin{array}{c}\text { Unpump } \\
\text { Time }\end{array}$ & 24 Hr Set \\
\hline 35 & & & & & $2: 00$ & hard \\
\hline 25 & & & & & $2: 20$ & hard \\
\hline 20 & & & & & $2: 40$ & hard \\
\hline 15 & & & & & $3: 00$ & hard \\
\hline 10 & & & & & $8: 00$ & soft \\
\hline 5 & & & & & over 8 & no set \\
\hline 5 & 15 & & & & $5: 20$ & haft \\
\hline 13 & 5 & & & & $6: 00$ & soft \\
\hline 15 & 5 & & & & $4: 00$ & hard \\
\hline 20 & 5 & & & & $4: 20$ & hard \\
\hline 25 & 5 & & & & $7: 00$ & hard \\
\hline 25 & 10 & & & & $5: 20$ & hard \\
\hline 20 & & 20 & & & $7: 00$ & hard \\
\hline 25 & & 20 & & & $4: 40$ & hard \\
\hline 25 & & 30 & & & & hard \\
\hline 30 & & 30 & & & & \\
\hline 40 & & 30 & & & & \\
\hline
\end{tabular}




\section{(8S) CSI Technologies}

120 Deg F Table - Percentage of Component to base Resin

\begin{tabular}{|c|c|c|c|c|c|c|}
\hline Comp A & Comp B & Comp C & Comp D & Comp E & $\begin{array}{c}\text { Unpump } \\
\text { Time }\end{array}$ & $24 \mathrm{Hr}$ Set \\
\hline 25 & & & & & $1: 40$ & hard \\
\hline 20 & & & & & $2: 00$ & hard \\
\hline 19 & & & & & $2: 40$ & hard \\
\hline 18 & & & & & 3:00 & hard \\
\hline 17 & & & & & $3: 20$ & hard \\
\hline 15 & & & & & $8: 00$ & hard \\
\hline 30 & 15 & & & & $2: 00$ & hard \\
\hline 30 & 10 & & & & $1: 40$ & hard \\
\hline 30 & 5 & & & & $1: 40$ & hard \\
\hline 30 & 5 & & & & $2: 20$ & hard \\
\hline 25 & 10 & & & & $5: 00$ & hard \\
\hline 25 & 5 & & & & $2: 00$ & hard \\
\hline 20 & 5 & & & & $5: 40$ & hard \\
\hline 15 & 10 & & & & 8:00 & hard \\
\hline 10 & 15 & & & & over 8 & hard \\
\hline 20 & & 20 & & & $5: 20$ & hard rubber \\
\hline 25 & & 20 & & & 4:00 & hard rubber \\
\hline 20 & & 30 & & & 9:00 & hard rubber \\
\hline 22 & & 30 & & & 8:00 & hard rubber \\
\hline 25 & & 30 & & & $5: 20$ & hard rubber \\
\hline 27 & & 30 & & & 5:00 & hard rubber \\
\hline 30 & & 30 & & & 4:00 & hard rubber \\
\hline 35 & & 30 & & & $3: 20$ & hard rubber \\
\hline 40 & & 30 & & & $3: 00$ & hard \\
\hline
\end{tabular}




\section{(영 CSI Technologies}

140 Deg F Table - Percentage of Component to base Resin

\begin{tabular}{|c|c|c|c|c|c|c|}
\hline Comp A & Comp B & Comp C & Comp D & Comp E & $\begin{array}{l}\text { Unpump } \\
\text { Time }\end{array}$ & $24 \mathrm{Hr} \mathrm{Set}$ \\
\hline 20 & & & & & $1: 20$ & hard \\
\hline 18 & & & & & $1: 40$ & hard \\
\hline 15 & & & & & 2:00 & hard \\
\hline 20 & 5 & & & & 1:40 & hard \\
\hline 20 & 10 & & & & $2: 00$ & hard \\
\hline 20 & 15 & & & & $2: 20$ & hard \\
\hline 20 & 20 & & & & $2: 20$ & hard \\
\hline 20 & 25 & & & & $2: 20$ & hard \\
\hline 15 & 5 & & & & $2: 20$ & hard \\
\hline 15 & 15 & & & & 5:00 & hard \\
\hline 15 & 25 & & & & $5: 20$ & hard \\
\hline 10 & 5 & & & & $4: 40$ & hard \\
\hline 10 & 15 & & & & 5:00 & hard \\
\hline 10 & 25 & & & & 5:20 & hard \\
\hline 5 & 10 & & & & 12:00 & tacky \\
\hline 5 & 15 & & & & 12:00 & tacky \\
\hline 5 & 20 & & & & 12:00 & tacky \\
\hline 15 & & 10 & & & $3: 20$ & hard \\
\hline 15 & & 20 & & & 6:00 & hard rubber \\
\hline 25 & & 20 & & & $2: 20$ & hard \\
\hline 15 & & 30 & & & 9:00 & hard rubber \\
\hline 20 & & 30 & & & 6:00 & hard rubber \\
\hline 22 & & 30 & & & 4:00 & hard rubber \\
\hline 25 & & 30 & & & $3: 20$ & hard \\
\hline 30 & & 30 & & & $2: 00$ & hard rubber \\
\hline 35 & & 30 & & & $1: 40$ & hard \\
\hline 40 & & 30 & & & $1: 20$ & hard \\
\hline
\end{tabular}

160 Deg F Table - Percentage of Component to base Resin

\begin{tabular}{|c|c|c|c|c|c|c|}
\hline Comp A & Comp B & Comp C & Comp D & Comp E & $\begin{array}{c}\text { Unpump } \\
\text { Time }\end{array}$ & 24 Hr Set \\
\hline 15 & 5 & & & & $1: 40$ & hard \\
\hline 15 & 15 & & & & $2: 20$ & hard \\
\hline 15 & 25 & & & & $2: 20$ & hard \\
\hline 15 & 35 & & & & $2: 40$ & hard \\
\hline 10 & 5 & & & & $3: 00$ & hard \\
\hline 10 & 15 & & & & $3: 00$ & hard \\
\hline 10 & 25 & & & & $3: 20$ & hard \\
\hline 10 & 35 & & & & $3: 40$ & hard \\
\hline 10 & 40 & & & & $4: 00$ & hard \\
\hline 10 & 45 & & & & $4: 00$ & hard \\
\hline 10 & 55 & & & & $2: 00$ & hard \\
\hline 15 & & 10 & & & $3: 40$ & hard rubber \\
\hline 15 & & 20 & & & $7: 40$ & hard rubber \\
\hline 15 & & 30 & & & $6: 40$ & hard \\
\hline & 30 & & & & $6: 20$ & hard \\
\hline & 35 & & & & $7: 20$ & hard \\
\hline & 45 & & & & & hard \\
\hline
\end{tabular}




\section{(बis) CSI Technologies}

180 Deg F Table - Percentage of Component to base Resin

\begin{tabular}{|c|c|c|c|c|c|c|}
\hline $\operatorname{Comp} A$ & Comp B & Comp C & Comp D & Comp E & $\begin{array}{l}\text { Unpump } \\
\text { Time }\end{array}$ & $24 \mathrm{Hr}$ Set \\
\hline 10 & 5 & & & & $2: 00$ & hard \\
\hline 10 & 15 & & & & 2:00 & hard \\
\hline 10 & 25 & & & & 2:00 & hard \\
\hline 10 & 35 & & & & 2:00 & hard \\
\hline 10 & 40 & & & & $2: 00$ & hard \\
\hline 15 & & 20 & & & $2: 40$ & hard \\
\hline 20 & & 20 & & & $1: 20$ & hard \\
\hline 25 & & 20 & & & $0: 40$ & hard \\
\hline \multirow[t]{10}{*}{30} & & 20 & & & $0: 20$ & hard \\
\hline & 15 & 20 & & & never hard & never hard \\
\hline & 25 & 20 & & & never hard & never hard \\
\hline & 15 & & & & never hard & never hard \\
\hline & 20 & & & & 7:00 & hard \\
\hline & 25 & & & & 5:20 & hard \\
\hline & 30 & & & & 4:40 & hard \\
\hline & 35 & & & & 4:00 & hard \\
\hline & 40 & & & & 3:40 & hard \\
\hline & 45 & & & & 3:40 & hard \\
\hline
\end{tabular}

200 Deg F Table - Percentage of Component to base Resin

\begin{tabular}{|c|c|c|c|c|c|c|}
\hline $\operatorname{Comp} A$ & Comp B & Comp C & Comp D & Comp E & $\begin{array}{l}\text { Unpump } \\
\text { Time }\end{array}$ & $24 \mathrm{Hr}$ Set \\
\hline & 20 & & & & $4: 00$ & hard \\
\hline & 25 & & & & $3: 20$ & hard \\
\hline & 30 & & & & $2: 40$ & hard \\
\hline & 35 & & & & $2: 20$ & hard \\
\hline & 40 & & & & $2: 20$ & hard \\
\hline & 45 & & & & $2: 20$ & hard \\
\hline & 20 & 10 & & & $5: 40$ & hard \\
\hline & 25 & 10 & & & $4: 20$ & hard \\
\hline & 30 & 10 & & & $3: 40$ & hard \\
\hline & 35 & 10 & & & $3: 40$ & hard \\
\hline & 20 & 20 & & & $8: 40$ & hard \\
\hline & 25 & 20 & & & 6:00 & hard \\
\hline & 30 & 20 & & & $5: 00$ & hard \\
\hline & 35 & 20 & & & $4: 20$ & hard \\
\hline
\end{tabular}




\section{(\$4) CSI Technologies}

220 Deg F Table - Percentage of Component to base Resin

\begin{tabular}{|c|c|c|c|c|c|c|}
\hline Comp A & Comp B & Comp C & Comp D & Comp E & $\begin{array}{c}\text { Unpump } \\
\text { Time }\end{array}$ & 24 Hr Set \\
\hline & 20 & 20 & & & $6: 20$ & hard rubber \\
\hline & 25 & 20 & & & $4: 00$ & hard rubber \\
\hline & 30 & 20 & & & $3: 00$ & hard \\
\hline & 35 & 20 & & & $2: 40$ & hard \\
\hline
\end{tabular}

240 Deg F Table - Percentage of Component to base Resin

\begin{tabular}{|c|c|c|c|c|c|c|}
\hline Comp A & Comp B & Comp C & Comp D & Comp E & $\begin{array}{c}\text { Unpump } \\
\text { Time }\end{array}$ & 24 Hr Set \\
\hline & 15 & 20 & & & $7: 30$ & hard rubber \\
\hline & 20 & 20 & & & $4: 30$ & hard \\
\hline & 25 & 20 & & & $3: 00$ & hard \\
\hline & 30 & 20 & & & $2: 00$ & hard \\
\hline & 35 & 20 & & & $2: 00$ & hard \\
\hline
\end{tabular}

The following sheets are thickening time and rheology tests conducted with the resin product formulated for $340 \mathrm{deg}$ F. 


\section{(बSi) CSI Technologies}

Resin Formulation:

100:40:17

Resin: Comp E: Comp B

Weighout

\begin{tabular}{|l|c|c|c|c|c|}
\hline Comp & sg & gms & cc & \% bwor & 150 gm mix \\
\hline Barite & 4.34 & 0 & 0 & 0 & 0 \\
\hline Resin & 1.19 & 414 & 348 & 100 & 96 \\
\hline Comp E & .96 & 165.6 & 173 & 40 & 38.4 \\
\hline Comp B & 1.02 & 70.4 & 69 & 17 & 16.3 \\
\hline $\begin{array}{l}\text { SF 325 } \\
\text { mesh }\end{array}$ & 2.65 & 0 & 0 & 0 & 0 \\
\hline
\end{tabular}

Thickening Time: at $340 \mathrm{deg}$ F, 1,850 initial, 12,200 psi reached in 65 minutes

14,480 ft Well, 340 deg F BHCT

Test \# 100-203

Initial Bc

3

$40 \mathrm{BC}$

$1: 25$

$70 \mathrm{BC}$

$1: 26$

Test tubes at $24 \mathrm{hr}$ :Firm / Soft

\section{Rheology}

\begin{tabular}{|l|c|c|}
\hline Speed & 80 deg F & 190 deg F \\
\hline 600 & & \\
\hline 300 & 190 & 24 \\
\hline 200 & 130 & 14 \\
\hline 100 & 68 & 6 \\
\hline 60 & 40 & 2 \\
\hline 30 & 20 & 1 \\
\hline 6 & 4 & 1 \\
\hline 3 & 2 & 1 \\
\hline PV & & \\
\hline TY & & \\
\hline
\end{tabular}




\section{(BS) CSI Technologies}

Resin Formulation:

100:40:17:10

Resin: Comp C: Comp B: Comp E

Weighout

\begin{tabular}{|l|c|c|c|c|c|}
\hline Comp & sg & gms & cc & \% bwor & 150 gm mix \\
\hline Comp E & .96 & 38.9 & 41 & 10 & \\
\hline Resin & 1.19 & 389 & 327 & 100 & \\
\hline Comp C & .99 & 155.6 & 157 & 40 & \\
\hline Comp B & 1.02 & 66.1 & 65 & 17 & \\
\hline $\begin{array}{l}\text { SF 325 } \\
\text { mesh }\end{array}$ & 2.65 & 0 & 0 & 0 & \\
\hline
\end{tabular}

Thickening Time: at $340 \mathrm{deg}$ F, 1,850 initial, 12,200 psi reached in 65 minutes

14,480 ft Well, 340 deg F BHCT

Test \#

111-389

Initial Bc

5

$40 \mathrm{Bc}$

$4: 58$

$70 \mathrm{BC}$

$5: 40$

Test tubes at $24 \mathrm{hr}$ :Soft

Rheology

\begin{tabular}{|l|c|c|}
\hline Speed & 80 deg F & 190 deg F \\
\hline 600 & 248 & 30 \\
\hline 300 & 130 & 12 \\
\hline 200 & 86 & 8 \\
\hline 100 & 44 & 4 \\
\hline 60 & 24 & 2 \\
\hline 30 & 12 & 1 \\
\hline 6 & 2 & 1 \\
\hline 3 & 1 & 1 \\
\hline PV & 243 & \\
\hline TY & 5 & \\
\hline
\end{tabular}




\section{(बSi) CSI Technologies}

Appendix B-Thickening Time Tests on Pre-Stressed Cement

Pre-Stressed Cement

Weighout

\begin{tabular}{|l|c|c|c|c|}
\hline Comp & Conc & unit & SG & Grams \\
\hline Lehigh H & 94 & lb / sk & 3.14 & 546.34 \\
\hline SA-1 & .5 & $\%$ bwoc & .6 & 2.73 \\
\hline SA-2 & 25 & \% bwoc & 3 & 136.59 \\
\hline SA-3 & 2.5 & \% bwoc & 1.43 & 13.66 \\
\hline SA-4 & 30 & \% bwoc & 3.58 & 163.90 \\
\hline SA-5 & .14 & gal/sk & 1.26 & 191.22 \\
\hline $\begin{array}{l}\text { 100 mesh } \\
\text { silica sand }\end{array}$ & 35 & \% bwoc & 2.65 & 8.55 \\
\hline $\begin{array}{l}\text { Fresh } \\
\text { Water }\end{array}$ & 5 & gal/sk & 1 & 242.37 \\
\hline
\end{tabular}

Density: $\quad 18.12$

Yield: $\quad 1.66$

Free Fluid

\begin{tabular}{|l|l|}
\hline Conditioning Time (hr:min): & $0: 20$ \\
\hline Measured (mL): & 0 \\
\hline Free Fluid $(\%):$ & 0 \\
\hline
\end{tabular}

Stirred Fluid Loss

\begin{tabular}{|l|l|}
\hline Conditioning Time (hr:min): & $0: 20$ \\
\hline Test Temp (deg F): & 230 \\
\hline Collected Fluid (mL): & 17 \\
\hline Time (min): & 30 \\
\hline API FL (mL/30 m): & 34 \\
\hline
\end{tabular}




\section{(B) CSI Technologies}

Thickening Time

\begin{tabular}{|l|c|c|}
\hline Test \# & $100-393$ & $100-582$ \\
\hline Test Temp (deg F) & 230 & 230 \\
\hline Time to Temp (min) & 68 & 68 \\
\hline Initial Pressure (psi) & 825 & 825 \\
\hline Final Pressure (psi) & 9825 & 9825 \\
\hline Initial Bc & 27 & 16 \\
\hline $40 \mathrm{Bc}$ (hr:min) & $3: 06$ & $3: 24$ \\
\hline $70 \mathrm{Bc}$ (hr:min) & $3: 37$ & $3: 48$ \\
\hline 100 Bc (hr:min) & & \\
\hline
\end{tabular}

Rheology
\begin{tabular}{|l|c|c|}
\hline Speed & 80 deg F & $190 \operatorname{deg}$ F \\
\hline 600 & & \\
\hline 300 & 366 & 132 \\
\hline 200 & 254 & 88 \\
\hline 100 & 130 & 42 \\
\hline 60 & 78 & 22 \\
\hline 30 & 38 & 10 \\
\hline 6 & 6 & 2 \\
\hline 3 & 4 & 1 \\
\hline PV & 354 & 135 \\
\hline TY & 12 & -3 \\
\hline
\end{tabular}




\section{(영 CSI Technologies}

Appendix C -Epoxy Resin Job Reports 


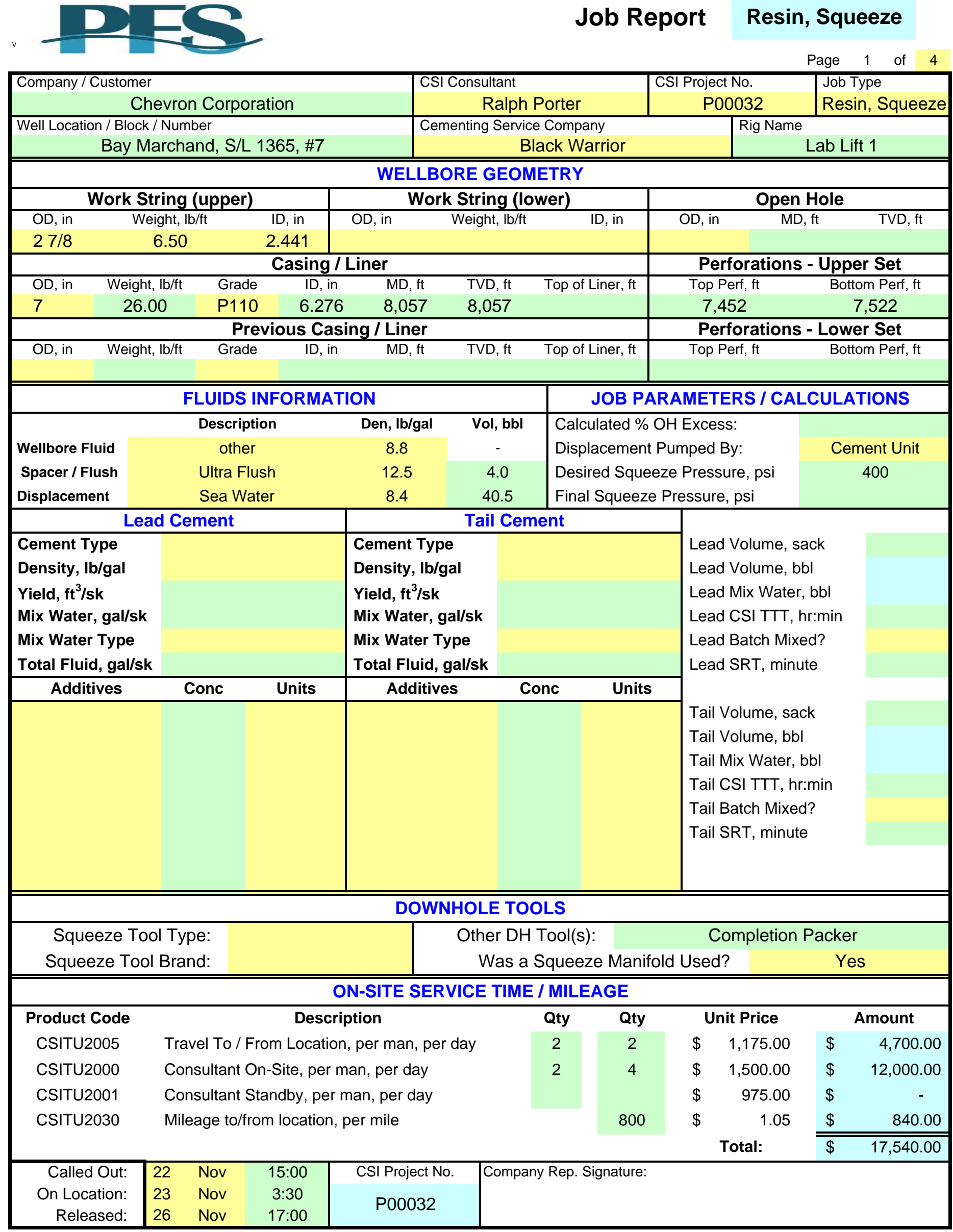


Component A of the Ultra Seal R. was very thick and took $2 \mathrm{hrs}$.to drain 2 tanks, This was found as unsatisfactory to the customer. Imediate attention must be addressed to this problem as the weather is turning cooler every day.

\begin{tabular}{|c|c|c|c|c|c|c|}
\hline \multicolumn{7}{|c|}{ JOB LOG } \\
\hline $\begin{array}{c}\text { Date } \\
\text { dd Month }\end{array}$ & $\begin{array}{c}\text { Time } \\
\text { hr:min }\end{array}$ & $\begin{array}{c}\text { Density } \\
\text { lb/gal }\end{array}$ & $\begin{array}{l}\text { Rate } \\
\text { bpm }\end{array}$ & $\begin{array}{c}\text { Pressure } \\
\text { psi }\end{array}$ & $\begin{array}{c}\text { Volume } \\
\text { bbl }\end{array}$ & Event \\
\hline 22 Nov & $15: 00$ & & & & & Called out \\
\hline 23 Nov & $3: 00$ & & & & & Arrive @ Chevron Dock, Leeville, La. \\
\hline & $3: 30$ & & & & & On Board Lift Boat Lift Lab \#1 \\
\hline & 7:00 & & & & & Orientation at Chevron Production Facility \\
\hline & $8: 30$ & & & & & JSA Meeting \\
\hline & 9:00 & & & & & Move Lift Boat over to load out dock \\
\hline & $12: 00$ & & & & & Start load out \\
\hline & $14: 00$ & & & & & Pump equipment on board Lab Lift \#1 Move Blue streak \#7 \\
\hline & & & & & & into position Continue Load Out chemicals \\
\hline & $15: 00$ & & & & & Lab Lift \#1 Having problems jacking down, Make repairs \\
\hline 24 Nov & $5: 30$ & & & & & Repairs complete, Depart dock for location \\
\hline & $7: 30$ & & & & & Arrive on Location, Position Lift boats \& Dive Boat \\
\hline & $8: 00$ & & & & & Rig up JSA \\
\hline & $8: 45$ & & & & & Start Rig up \\
\hline & $12: 00$ & & & & & Divers rig up on Tbg \& Annulus \\
\hline & $15: 00$ & & & & & Control Line to short, Order additional Control Line \\
\hline & $16: 05$ & & & & & Start test lines against crown valve to $1500 \mathrm{psi}$ \\
\hline & $16: 39$ & & & & & Test good, Test lines to csg. Valve. \\
\hline & $16: 53$ & & & & & Test good, Test lines to csg. Valve. \\
\hline & $17: 00$ & & & & & Run Control line for SSCV \\
\hline & $17: 49$ & & & & & Open well on Tbg. \& Csg., Open SSCV \\
\hline & 18:07 & & 1.5 & 400 & & Pump down TBG W/ Dyed water, Check for Tbg. Leaks \\
\hline & $18: 14$ & & & & 5.0 & Shut down, Diver not in position to check for leaks \\
\hline & & & & & & Confirmed SSCV open \\
\hline & $18: 35$ & & 1.0 & 400 & & Diver in position to check for leaks, Pump down Tbg. \\
\hline & $18: 37$ & & & & 2.0 & Shut down, No leaks seen \\
\hline & $18: 40$ & & 1.0 & 200 & & Check for leaks \\
\hline & $18: 48$ & & & & 7.0 & Leak in Csg. Split, Shut down \\
\hline & $18: 57$ & & 1.0 & 400 & & Pump down TBG W/ Dyed water, \\
\hline & 19:00 & & & & 2.0 & Shut down dyed water through Csg. Split, Hydraulic problem \\
\hline & & & & & & on pump unit \\
\hline & 19:56 & & & & & Problem corrected \\
\hline & $20: 14$ & & 1.0 & 400 & & Pump down tbg.w/ dyed water \\
\hline
\end{tabular}




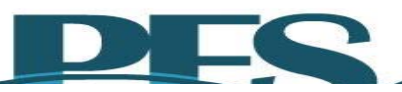

Job Report Resin, Squeeze

Page 3 of 4

\begin{tabular}{|c|c|c|c|c|c|c|}
\hline \multicolumn{3}{|c|}{ Bay Marchand, S/L 1365, \#7 } & \multicolumn{4}{|r|}{ CSI Project No. - P00032 } \\
\hline $\begin{array}{c}\text { Date } \\
\text { dd Month }\end{array}$ & $\begin{array}{c}\text { Time } \\
\text { hr:min }\end{array}$ & $\begin{array}{c}\text { Density } \\
\text { lb/gal }\end{array}$ & $\begin{array}{l}\text { Rate } \\
\text { bpm }\end{array}$ & $\begin{array}{c}\text { Pressure } \\
\text { psi }\end{array}$ & $\begin{array}{c}\text { Volume } \\
\text { bbl }\end{array}$ & Event \\
\hline \multirow[t]{2}{*}{24 Nov } & $20: 18$ & & & & 2.0 & Diver confirmed, Dyed water back out Csg. Split \\
\hline & & & & & & Shut down for night \\
\hline \multirow[t]{43}{*}{$25 \mathrm{Nov}$} & 8:00 & & & & & Wait on divers. \\
\hline & $8: 45$ & & & & & Diver in water \\
\hline & $8: 55$ & & & & & Swap Manifold over from Tbg. To Csg., Unsuspected psi \\
\hline & & & & & & on Tbg. Dumped on Csg. Close in Manifold, Isolate Tbg. Psi \\
\hline & & & & & & release pressure from pump, Open to Csg. Side \\
\hline & 9:13 & & & 230 & & Pressure on Tbg., Pump down Csg \\
\hline & 9:22 & & & 200 & 15.0 & Clear Csg. From Dye \\
\hline & $10: 29$ & & & & 23.0 & Finish Pump Down Csg. \\
\hline & $10: 47$ & & 2.0 & 380 & & Pump down Tbg. W/ Dyed water, Diver check for Tbg. Leaks \\
\hline & $10: 53$ & & 2.0 & 375 & 7.0 & Shut down, Open SSCV \\
\hline & $11: 07$ & & 2.0 & 280 & & Pump down Tbg. \\
\hline & $11: 11$ & & & 200 & 14.0 & Shut down monitor pressure \\
\hline & $11: 13$ & & & 180 & & \\
\hline & $11: 23$ & & 2.0 & 250 & & Start pump down Tbg. Dyed water \\
\hline & $11: 29$ & & 2.0 & 220 & 24.0 & Shut down monitor pressure \\
\hline & $11: 30$ & & & 175 & & \\
\hline & $12: 03$ & & 2.0 & 200 & & Resume pump down Tbg. Dyed water \\
\hline & $12: 10$ & & 2.0 & 180 & 34.0 & Bubbles in water no dye back \\
\hline & $12: 11$ & & & & & Shut down observe Bubbles, Wait on diver to get in water \\
\hline & $13: 00$ & & & & & Decreasing bubble, Diver down \\
\hline & $13: 13$ & & & 50 & & Start pump down Tbg. Dyed water \\
\hline & 13:17 & & 2.0 & 225 & 5.0 & Start pump down Tbg. Dyed water, Diver monitor bubbles \\
\hline & & & & & & through Csg. Split, No dye back some oil \\
\hline & $13: 57$ & & 2.0 & 100 & 70.0 & Shut down, Bubbles continue no dye back \\
\hline & $14: 05$ & & & & & JSA for mixing and pumping Resin squeeze \\
\hline & $15: 10$ & & & & & Start mix 12\# Ultra Sweep Spacer \\
\hline & $15: 47$ & & & & & Add Barite to $12 \# 4$ bbls. \\
\hline & $15: 55$ & & & & & Blend spacer while wait for Displacement tanks to fill \\
\hline & $16: 39$ & & 1.0 & & & Displacement tanks full Start pump Spacer \\
\hline & $16: 45$ & & 0.5 & & 4.0 & Finish pump spacer, Wash up, Drain \& dry equipment \& lines \\
\hline & $17: 20$ & & & & & Start mix Ultra Seal R. Component A, Resin draining very \\
\hline & & & & & & slow \\
\hline & $17: 50$ & & & & & Start add second Tote of Component A \\
\hline & 19:15 & & & & & Finish Component A, Start add Component B \\
\hline & $19: 22$ & & & & & Component B. in, Blend Ultra Seal R, 10 BBls. \\
\hline & 19:36 & & 2.0 & 350 & & Start pump Ultra Seal R. \\
\hline & $19: 43$ & & 0.5 & & 10.0 & Finish pump Ultra Seal Start Displacement \\
\hline & 19:58 & & 1.8 & 250 & 24.0 & \\
\hline & $20: 04$ & & 1.5 & 230 & 34.0 & Spacer at perfs, slight increase in Pressure \\
\hline & $20: 07$ & & 1.0 & 200 & 37.0 & Ultra Seal R. at perfs, Decrease rate \\
\hline & $20: 09$ & & 0.5 & 180 & 40.0 & Decrease rate \\
\hline & $20: 10$ & & & 180 & 40.5 & Shut down \\
\hline & $20: 15$ & & & & & 5 minute pressure 0 psi \\
\hline
\end{tabular}




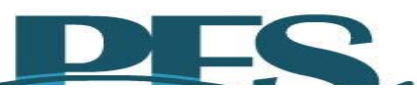

Job Report

Resin, Squeeze

Page 4 of 4

\begin{tabular}{|c|c|c|c|c|c|c|}
\hline \multicolumn{3}{|c|}{ Bay Marchand, S/L 1365, \#7 } & \multicolumn{4}{|r|}{ CSI Project No. - P00032 } \\
\hline $\begin{array}{c}\text { Date } \\
\text { dd Month }\end{array}$ & $\begin{array}{c}\text { Time } \\
\text { hr:min }\end{array}$ & $\begin{array}{c}\text { Density } \\
\mathrm{lb} / \mathrm{gal}\end{array}$ & $\begin{array}{l}\text { Rate } \\
\text { bpm }\end{array}$ & $\begin{array}{c}\text { Pressure } \\
\text { psi }\end{array}$ & $\begin{array}{c}\text { Volume } \\
\text { bbl }\end{array}$ & Event \\
\hline \multirow[t]{3}{*}{25 Nov } & $20: 20$ & & & & & Dump Methanol Tote in Blender, Start wash up \\
\hline & 21:05 & & & & & Finish wash up place used wash up methanol tote back on \\
\hline & & & & & & Blue streak 7, Lock in Tbg. 0 psi over night \\
\hline \multirow[t]{9}{*}{26 Nov } & $7: 00$ & & & & & Pressure on manifold 0, no bubbles, no slick visible \\
\hline & $7: 30$ & & & & & Morning JSA \\
\hline & $8: 20$ & & & 400 & 3.0 & Pressure up down Tbg. To test resin squeeze plug. \\
\hline & & & & & & Slight bubbles @ surface, Shut down Pressure bleed to 0 \\
\hline & & & & & & Plug Squeeze considered successful \\
\hline & $11: 00$ & & & & & Pump to flush flow line \\
\hline & $11: 35$ & & & & & Finish Flush, Start rig down \\
\hline & & & & & & Jack Down \& sail to Leeville,La. \\
\hline & $17: 00$ & & & & & On beach, CSI released \\
\hline & & & & & & \\
\hline & & & & & & \\
\hline & & & & & & \\
\hline & & & & & & \\
\hline & & & & & & \\
\hline & & & & & & \\
\hline & & & & & & \\
\hline & & & & & & \\
\hline & & & & & & \\
\hline & & & & & & \\
\hline & & & & & & \\
\hline & & & & & & \\
\hline & & & & & & \\
\hline & & & & & & \\
\hline & & & & & & \\
\hline & & & & & & \\
\hline & & & & & & \\
\hline & & & & & & \\
\hline & & & & & & \\
\hline & & & & & & \\
\hline & & & & & & \\
\hline & & & & & & \\
\hline & & & & & & \\
\hline & & & & & & \\
\hline & & & & & & \\
\hline & & & & & & \\
\hline & & & & & & \\
\hline & & & & & & \\
\hline & & & & & & \\
\hline & & & & & & \\
\hline & & & & & & \\
\hline & & & & & & \\
\hline & & & & & & \\
\hline
\end{tabular}




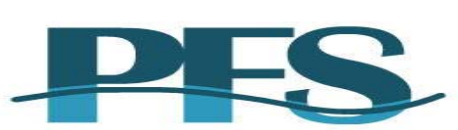

Resin Job Report

Job Type

Perf Squeeze

Company / Customer

Stone Energy

On Site Operator Supervisor

John Vincent
Well Location/Block or Field

Rig Name
Page 1 of 3

Number OCS-G

PFS Technicians

Glenn Morris

General Job Information

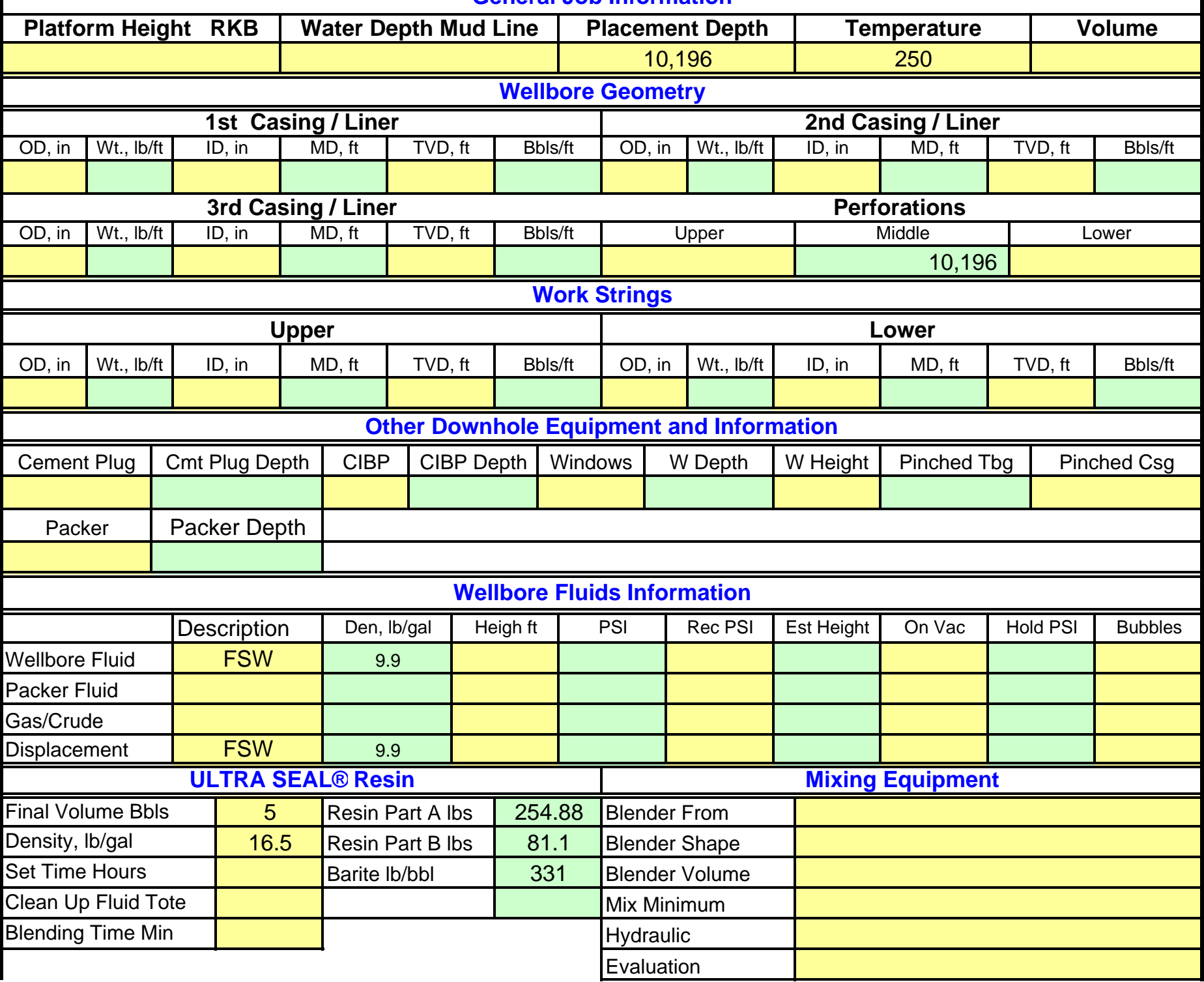

Comments and Observations

Job was to repair leaking perforations after prior cement squeeze failed. Tubing used for pump down.

$9.9 \mathrm{lb} / \mathrm{gal}$ barely controlled the well - job was bullheaded in while holding pressure on csg-tbg annulus. 


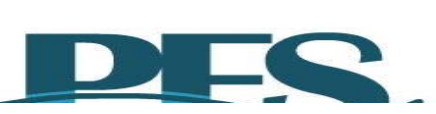

Resin Job Report

Job Type

Perf Squeeze

Page 2 of 3

\begin{tabular}{|c|c|c|c|c|c|c|}
\hline \multicolumn{7}{|c|}{ Job Log } \\
\hline \begin{tabular}{c|} 
Date \\
dd Month
\end{tabular} & $\begin{array}{c}\text { Time } \\
\text { hr:min }\end{array}$ & $\begin{array}{c}\text { Density } \\
\text { |b/gal }\end{array}$ & $\begin{array}{l}\text { Rate } \\
\text { bpm }\end{array}$ & $\begin{array}{c}\text { Pressure } \\
\text { psi }\end{array}$ & 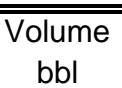 & Event \\
\hline 18 Mar & $10: 00$ & & & & & Called Out \\
\hline & $20: 00$ & & & & & At Dock \\
\hline 19 Mar & $7: 30$ & & & & & Arrive at Rig \\
\hline & 8:30 & & 2.0 & $\begin{array}{c}2,000 / \\
1,800\end{array}$ & & $\begin{array}{l}\text { Establish injection rate } 2 \text { bpm at 2,000 psi on tbg, 1,800 psi } \\
\text { on csg }\end{array}$ \\
\hline & 9:00 & & & & & Start mixing resin \\
\hline & $10: 20$ & & & & & Add resin part $A$ \\
\hline & $10: 30$ & & & & & Start adding barite \\
\hline & $11: 05$ & & 16.5 & & & Stop barite - 26 sks \\
\hline & $11: 20$ & & & & & Start adding resin part B \\
\hline & $11: 25$ & & & & & All part $B$ in, mix 5 to 10 minutes \\
\hline & $11: 35$ & & & & & Rig up 3" pump to feed triplex \\
\hline & $11: 40$ & & & & & Pump to triplex \\
\hline & $11: 45$ & & & $\begin{array}{c}2,200 / \\
1,550\end{array}$ & & Switch to displacement \\
\hline & $11: 55$ & & & \begin{tabular}{c|c|}
$2,200 /$ \\
1,550
\end{tabular} & 20.0 & $20 \mathrm{bbl}$ displacement gone \\
\hline & $12: 00$ & & & & 25.0 & $25 \mathrm{bbl}$ displacement gone \\
\hline & $12: 03$ & & & \begin{tabular}{|l|}
$2,200 /$ \\
1,500
\end{tabular} & 30.0 & $30 \mathrm{bbl}$ displacement gone \\
\hline & $12: 08$ & & & $\begin{array}{c}2,200 / \\
1,500\end{array}$ & 40.0 & $40 \mathrm{bbl}$ displacement gone \\
\hline & $12: 18$ & & & & & $\begin{array}{l}\text { Shut in - 2,050 psi on csg, 2,100 psi on tbg. Hold pressure } \\
14 \text { hours }\end{array}$ \\
\hline 20 Mar & $2: 30$ & & & & & Pressure test to 2,000 psi - pressure held, job complete \\
\hline & & & & & & \\
\hline & & & & & & \\
\hline & & & & & & \\
\hline & & & & & & \\
\hline & & & & & & \\
\hline & & & & & & \\
\hline & & & & & & \\
\hline & & & & & & \\
\hline & & & & & & \\
\hline & & & & & & \\
\hline & & & & & & \\
\hline & & & & & & \\
\hline & & & & & & \\
\hline & & & & & & \\
\hline & & & & & & \\
\hline
\end{tabular}




\section{(बSi) CSI Technologies}

Appendix D-Phase II Financials 
Phase II Budget

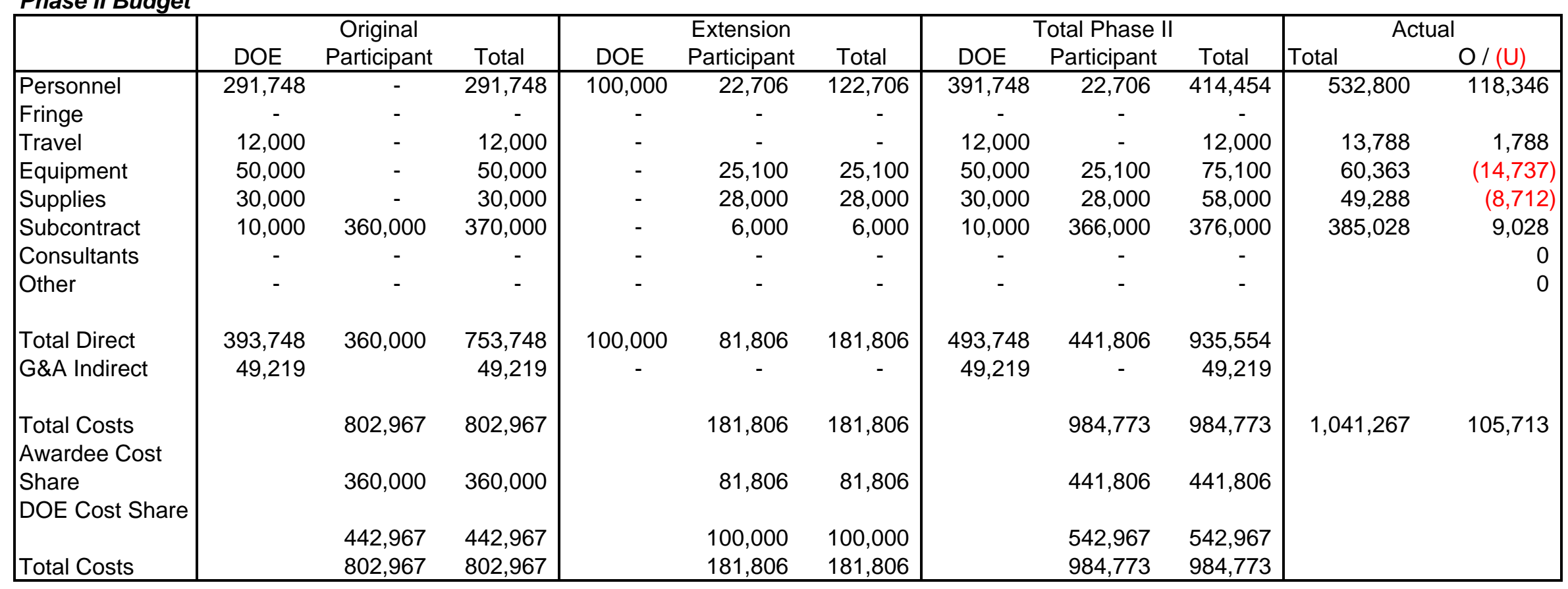

